\title{
Methods for quantitative study of divertor heat loads on W7-X
}

\author{
Yu Gao ${ }^{1}$, Marcin W. Jakubowski ${ }^{2,3}$, Peter Drewelow ${ }^{2}$, Fabio \\ Pisano $^{4}$, Aleix Puig Sitjes ${ }^{2}$, Holger Niemann ${ }^{2}$, Adnan $\mathrm{Ali}^{2}$, \\ Barbara Cannas ${ }^{4}$, and W7-X team ${ }^{1}$ \\ ${ }^{1}$ Forschungszentrum Jülich GmbH, institut für Energie- und \\ Klimaforschung - Plasmaphysik, Partner of the Trilateral \\ Euregio Cluster (TEC), 52425 Jülich, Germany \\ ${ }^{2}$ Max-Planck-Institut für Plasmaphysik, 17491 Greifswald, \\ Germany \\ ${ }^{3}$ University of Szczecin, Institute of Physics, 70-453 Szczecin, \\ Poland \\ ${ }^{4}$ University of Cagliari, Department of Electrical and \\ Electronic Engineering, 09123 Cagliari, Italy
}

February 28, 2019

\begin{abstract}
The paper presents procedures which have been developed for a quantitative analysis of the divertor power deposition at Wendelstein $7-\mathrm{X}$. The development of these tools is motivated by the need to compare and verify scientific and engineering predictions with experimental measurements. The measurements have been performed by means of the thermographic diagnostic system, capable of exploring the divertor heat loads, with the aim to study the heat load symmetry, compare footprint patterns with theoretical expectations, but also investigate leading edges and divertor misalignment. In order to compare measurements and numerical calculations, an accurate mapping between the camera data, the divertor geometry and the 3D CAD models has been constructed. This mapping allows to find a correspondence between the data in different representations, simplifying
\end{abstract}


data interpolation and visualization. This also provides a high resolution model of the target surface to compare numerical heat deposition calculations with experimental results from different cameras.

\section{Introduction}

For long pulse experiments in fusion devices, adequate evaluation of heat fluxes to plasma facing components is of vital importance. With the intrinsic three dimensional (3D) magnetic topology in Wendelstein 7-X (W7-X), more sophisticated methods are required to analyze the toroidally asymmetric power loads compared with the methods used in present day tokamaks.

$\mathrm{W} 7-\mathrm{X}$ is one of the largest (plasma volume $30 \mathrm{~m}^{3}$, magnetic major radius $R=5 \mathrm{~m}$, minor radius $a=0.5 \mathrm{~m}$ ) and the most advanced stellarators in the world so far $[1,2]$. The device is designed to demonstrate the capabilities of high plasma heating power $(10 \mathrm{MW})$ steady-state operations with reactorrelevant plasma parameters at pulse length up to 30 minutes [3]. The island divertor concept [4] (firstly used in W7-AS), was implemented in W7-X for operations phase 1.2a (OP1.2a) in the second half of 2017 [5,6]. Ten inertially cooled fine-grain graphite test divertor units (TDUs) are installed in the device with the divertor shape identical to the water-cooled divertor prepared for OP2.

W7-X has a five-fold modular machine symmetry, with flexible magnetic configurations generated by 70 superconducting magnetic coils. Each machine module consists of two half modules, each containing one TDU, as can be seen in Figure 1. Pre-defined helical magnetic island chains at the plasma boundary are cut open by the divertor plates, so that these open field lines lead the unconfined plasma to the graphite tiles.

Infrared thermography systems have been developed in many fusion devices as a key diagnostic for monitoring the surface temperatures and for studying the heat loads onto plasma facing components, and to prevent them from damage $[8,9,10,11,12,13]$. Due to the intrinsic nature of 3D distribution of heat loads in stellarators, it is essential to monitor the whole divertor plates by infrared cameras, for the safety of the experimental exploration aimed at high performance long discharges. In OP1.2, ten thermographic systems $[14,15]$ were installed for the first time, with each infrared camera monitoring one half module of the machine, realized by the wide-angle optics.

In addition to being one of the operational diagnostics for the safety of the machine [16], the high spatial resolution at the divertor targets from the camera view enables detailed studies of the divertor thermal footprints, which could provide important inputs for the following scientific and engineering 


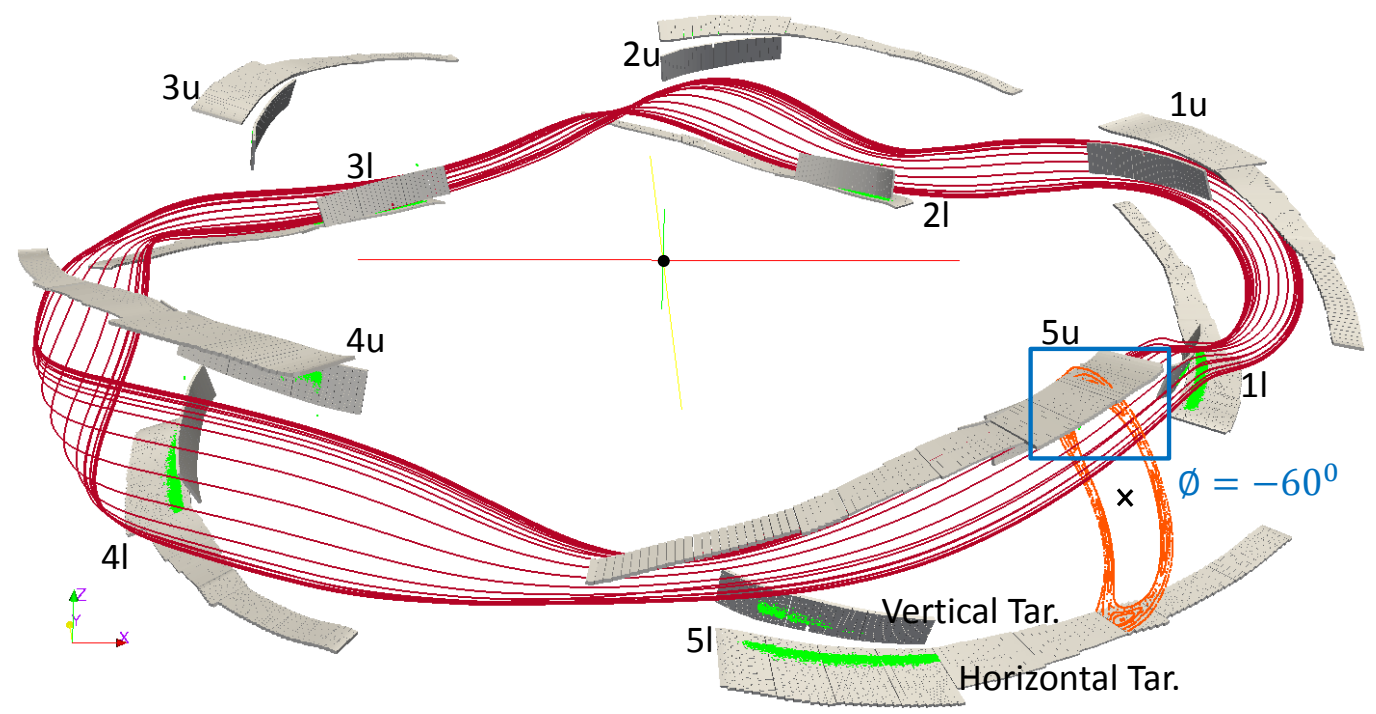

Figure 1: Overview of the island divertor in W7-X. The trajectory of a magnetic field line (red) in the ideal standard magnetic configuration is visualized. Ten divertor units (grey) are distributed toroidally with five in the upper and five in the lower part of the machine. Each divertor unit consisting of a horizontal and a vertical targets is labeled as 1-5 upper (u) or lower (l) targets. In the ideal standard magnetic configuration the power loads (green) are equally distributed to the ten divertor units, simulated by the heat diffusive modelling [7]. The calculated results from field line tracer in the scrape-off layer at a cross section of toroidal angle $\phi=-60^{\circ}$ will be presented in detail in Figure 2. The machine center is marked with black dot as the origin point for the 3D Cartesian coordinate, and the position of the magnetic axis at one cross section is marked with black cross. 
tasks:

\section{Power balance in 3D boundary.}

During a steady-state operation in magnetically confined plasma, the continuous loss of energy from the plasma is compensated by the externally supplied power and the $\alpha$-particle heating though collisional interactions with the plasma: $P_{\text {heat }}+P_{\alpha}=P_{\text {rad }}+P_{\text {sep }}$, where $P_{\text {rad }}$ is the power loss by radiation and $P_{\text {sep }}$ the unconfined power crossing over the separatrix. While it is true for both tokamak and stellarator equipped with divertor, that the dominant contribution for $P_{\text {sep }}$ is the total power flux $P_{\text {div }}$ guided onto the divertor plates, the methods to calulated $P_{\text {div }}$ has to be treated differently. In present tokamaks $P_{\text {div }}$ is often estimated with the assumption of toroidal symmetry, i.e. $P_{\mathrm{div}}=2 \pi R P_{\mathrm{pol}}$, where $P_{\text {pol }}$ represents the integrated power loads in one poloidal cross section [17]. This is not true for a 3D magnetic boundary like in W7-X or tokamaks with magnetic perturbations, where a complete calculation of the heat flux on the whole divertor is required. Having such accurate total divertor power measurements can also be used to benchmark the global radiation measurements, which are located presently in limited toroidal angles in W7-X.

2. The study of magnetic topology and the heat transport inside the boundary islands.

Typically in devices with 3D boundary a comparison between the measured thermal patterns and the modelled magnetic footprints on the targets is performed. The simulated results from the field line tracing include the connection length of the open field lines or the heat fluxes simulated considering diffusive heat transport at the stochastic edge $[7,18]$. Such comparisons under different magnetic configurations and plasma parameters would contribute to a better understanding of the heat transport and radiation inside the island divertor and are essential for the experimental exploration of an optimized operation regime for $\mathrm{W} 7-\mathrm{X}$.

\section{The symmetrisation of the target heat loads.}

A quantitative analysis and comparison of the divertor power loads in different half modules are required for the task of target heat loads symmetrisation. This would be important for high performance long pulse operation to avoid overloading of specific targets. The geometry of the ten half modules is designed ideally to be identical, and a quasi equally distributed power onto each half module is expected, with optimized error field corrections, to support the steady-state operations. 
In W7-X, error fields, as one source of the asymmetry, caused by the slight misalignment and deformations of the superconducting coils, has been studied in the limiter configuration [19, 20]. The assembly and building errors of the divertor targets could be another source resulting the asymmetry of power loads. Experimentally the complete set of the new thermographic systems for the island divertor configuration has the potential to judge the level of symmetry among half modules operated at different perturbative fields.

\section{Leading edges and misalignments.}

The target surfaces of W7-X are designed to avoid leading edges. But due to technical boundary conditions, small misalignment of the target components is inevitable. The actual gap heights in between target elements or the so called 'fingers' are measured before and after the campaign. The comparisons between modeling [21] and experimental results are important for the preparation of the water-cooled high heat flux divertor planned for the next campaign $(\sim 2020)$. It requires a development of methods for detailed power load distributions to resolve the thermal footprint at each target element. Finger dependent heat distributions at the edges for certain target elements have been observed throughout the experiments.

In order to fulfill the requirements of the above mentioned tasks, methods for quantitative analysis of divertor heat loads were developed and discussed in this paper. In section 2, we introduce the basic design of the island divertor as well as the developed overview thermography systems in W7-X. In section 3, a two dimensional (2D) projection method will be presented, which is developed as platform for visualization and comparison of the target surface temperature and heat loads in different half modules. A grid mapping is also developed and will be presented in section 4 for the interpolation of the scattered camera data into the fixed grid points. Lines of analysis defined inside each finger will be described, as a preparation for the calculation of the heat flux profiles. Finally, we will show a subdivision of the target in section 5, which provides the surfaces with high spatial resolution for the results of the heat diffusive simulation. Together with the same interpolation methods described in section 4 , a direct comparison between experimental results and simulations is possible in $2 \mathrm{D}$ planes. 


\section{2 island divertor and Thermography}

The main magnetic configurations are characterized with edge rotational transform $t_{a}=5 / m=5 / 6$ (low iota configuration), $5 / 5$ (standard and high mirror configuration), $5 / 4$ (high iota configuration), where $\mathrm{m}$ is the poloidal mode number [22]. In standard and high mirror magnetic configurations, five island chains are independent from each other, while in high and low iota configurations a single helical island flux tube is around the torus. Different magnetic configurations result in a variety of divertor thermal footprints. In ideal low iota magnetic configuration, only the low iota part of the divertor receives high heat flux, while the high iota magnetic configuration would deposit most of the power loads to the high iota tail $[5,6]$ (see Figure 3 for the different parts of the divertor).

The transport of the power loads to the divertor targets in W7-X, depends largely on the edge island structures. The typical target to target connection length in the island divertor in W7-X is by one order of magnitude longer than that in medium sized tokamak, which is determined by the small internal pitch angle of field line inside the edge island [18], $\Theta=r_{\mathrm{i}} t^{\prime} a / R \sim 0.001$, where $r_{\mathrm{i}}$ is island width, $t^{\prime}$ the internal shear of the island flux surface, $a$ the minor and $R$ the major radius. The open field line traced in the vicinity around the $\mathrm{X}$ point (separatrix), has the longest connection length and resides on the outermost contour of the island chain intersecting the target, as shown in Figure 2. The typical connection length profile on the target is unique according to the 3D island boundary geometry, which has a sharp increase from the private flux region, a quasi exponential decay and a sudden cut-off towards the other end of the target. The intrinsic long connection length in $\mathrm{W} 7-\mathrm{X}$ is beneficial for a broader wetted area on the target supported with active cooling at the edge [18].

Nine immersion tubes and one endoscope system are installed and monuted with infrared cameras to monitor the surface temperature of the in-vessel components, including all ten divertor units [14]. A scene model has been implemented to simulate the distortion from the wide-angle optics by three dimensional ray tracing from the focal point of the camera sensor to the invessel components, such that for each pixel in the 2D image a 3D Cartesian coordinate of the intersection point of the machine is assigned [23]. By fitting the reference points of the computer aided design (CAD) model of the vessel with the camera view, a pinhole camera model could be derived, including the effect of the fish-eye lens distortion. A distorted view of the CAD model has been thus produced and overlaid by the measurements, as shown in Figure 3. The boundary of different parts of the divertor, e.g. low iota, high iota tail are marked with different colors. 


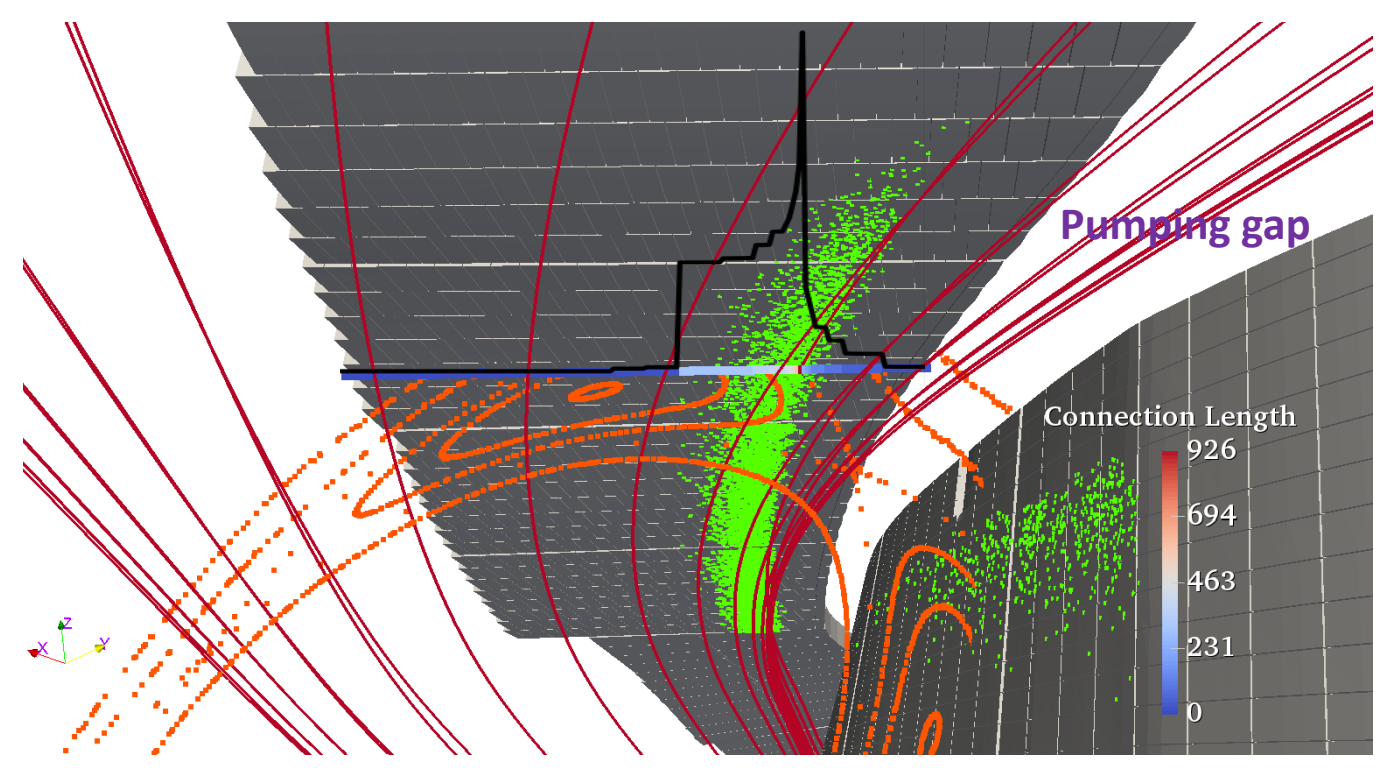

Figure 2: Field line tracing modelling in the scrape-off layer of the island divertor. The island topology at the edge is depicted with a Poincaré plot (orange), which is intersected by both horizontal and vertical targets. On the horizontal line of intersection, the target to target connection length profile of the field lines is rendered with a color map, and plotted in black. The open field line with the longest connection length close to the separatrix is traced (red). The diffusive field-line tracing simulates the footprints of power depositions, with the green dots representing the intersection points of the test particles to the target, traced from the last closed flux surface with self-defined diffusive coefficients. 


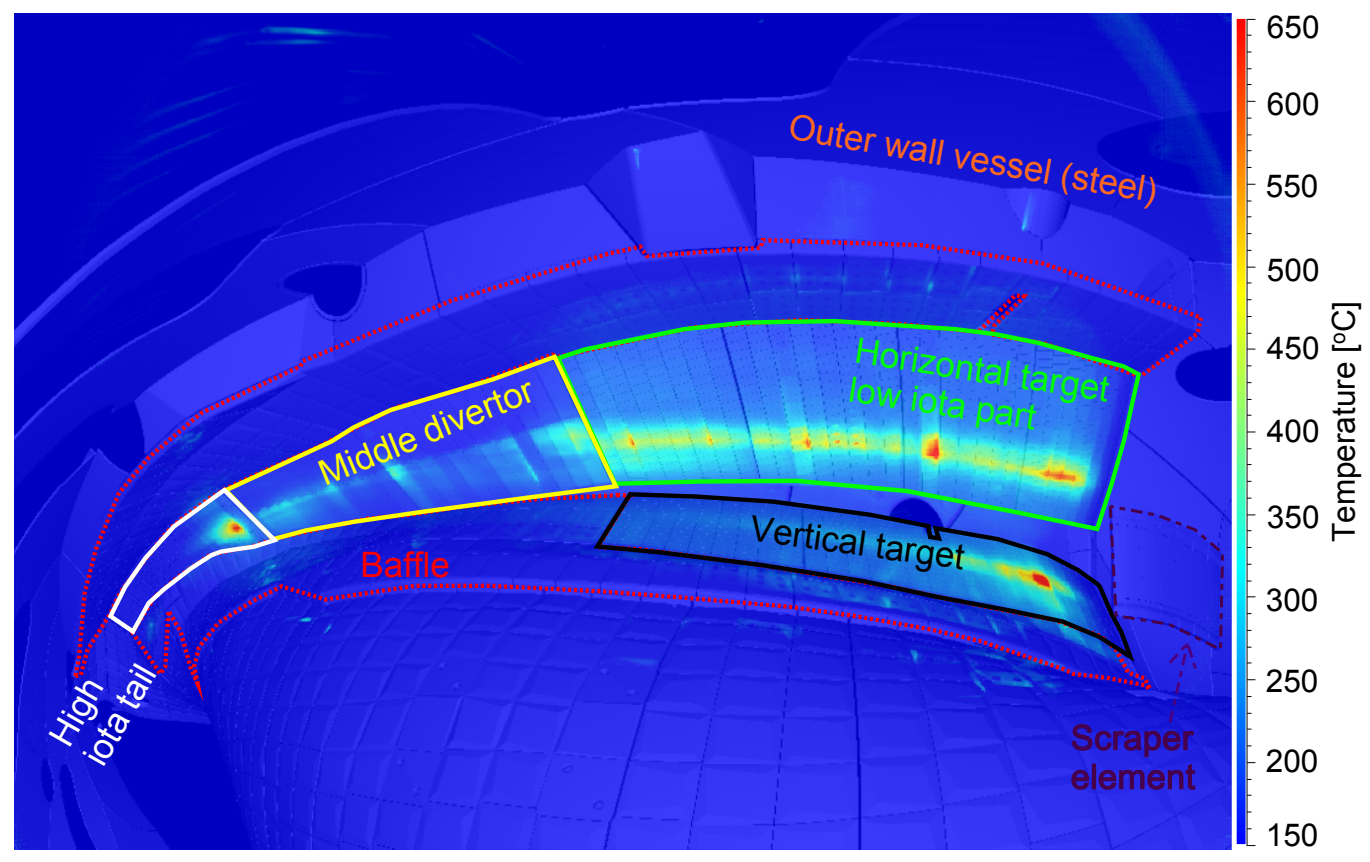

Figure 3: The distorted CAD model of the vessel overlaid by the surface temperature measured during the experiment with standard magnetic configuration from one of the infrared camera mounted on the immersion tube.

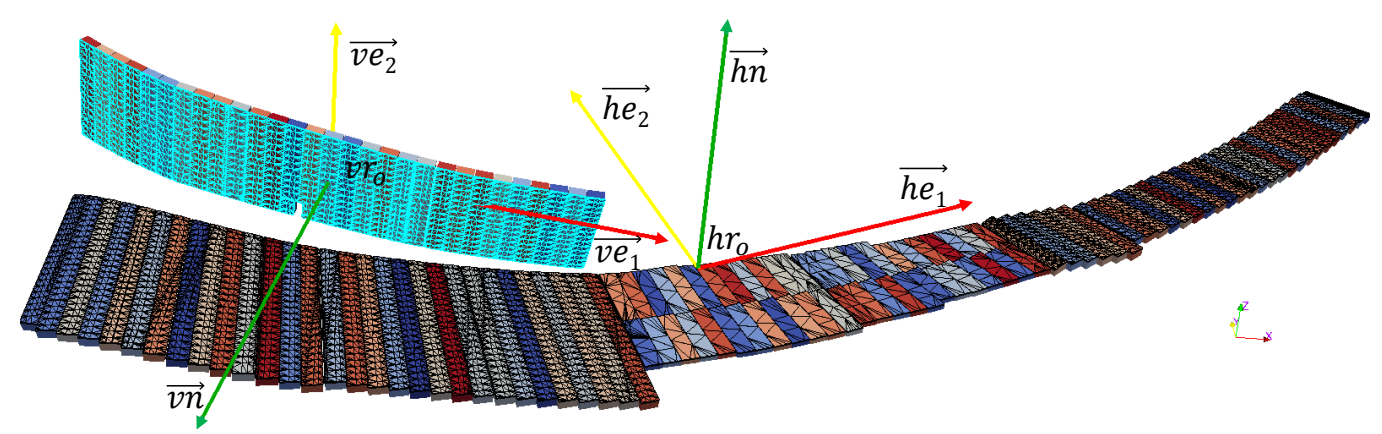

Figure 4: The extraction of the divertor surface from the CAD model and the vectors describing the $2 \mathrm{D}$ planes to be projected onto from the origin point $r_{o}$ for both horizontal $(\mathrm{h})$ and vertical $(\mathrm{v})$ targets. Normal vectors $\vec{n}$ in green, two axes direction $\overrightarrow{e_{1}}, \overrightarrow{e_{2}}$ in red and yellow. The connected blocks marked with different colors are the thermal insulated target elements or the so called divertor fingers. 

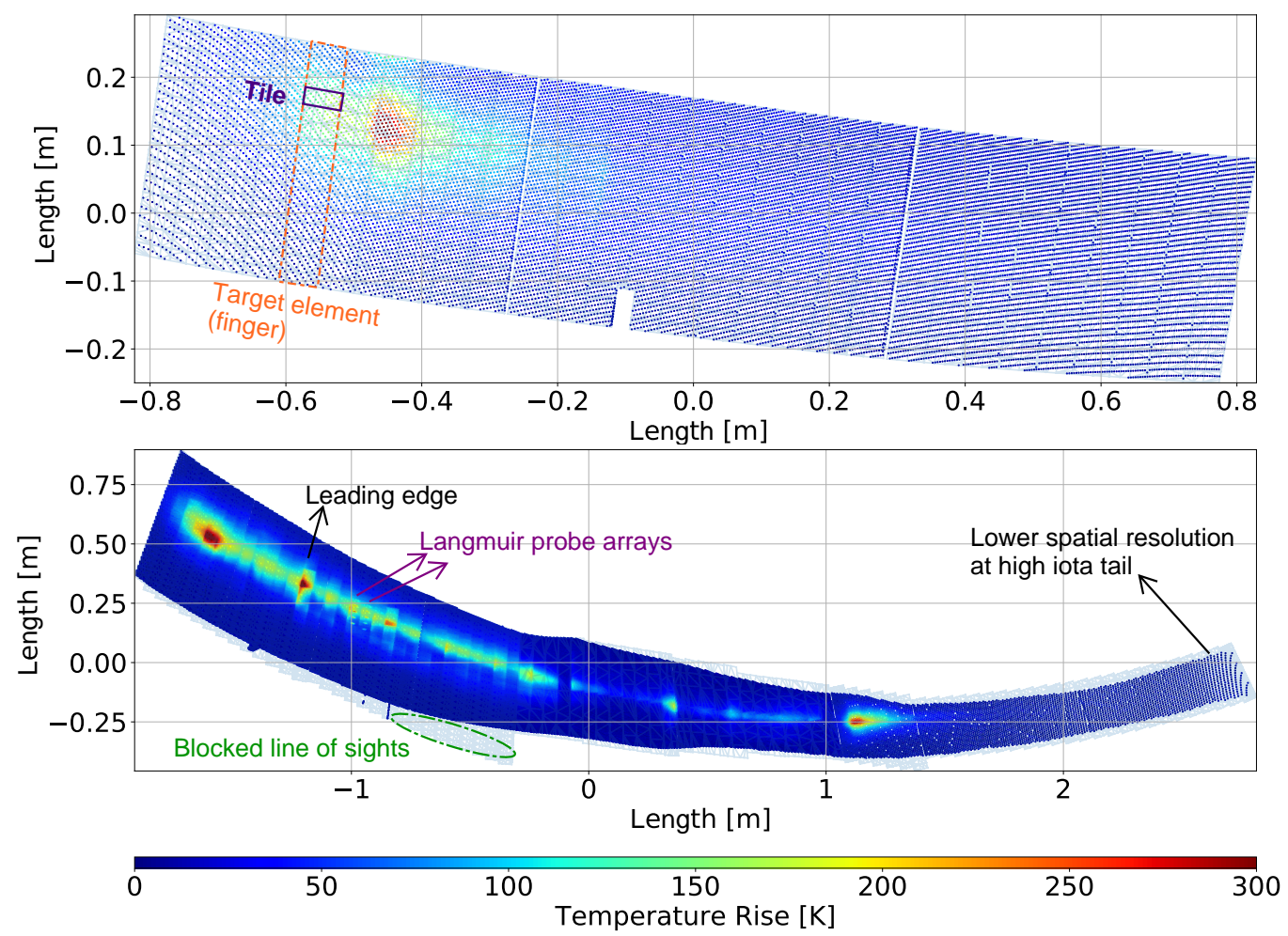

Figure 5: Projected camera data on the vertical (top figure) and horizontal (bottom figure) target with the divertor surface as the background. 
The infrared micro-bolometric cameras mounted on the immersion tubes were controlled to record the thermal emissions with a frame rate of $100 \mathrm{~Hz}$ in a wavelength range of $8-10 \mu \mathrm{m}$. The sensor array consists of $768 \times 1024$ pixels, with a varied spatial resolution at different parts of the divertor surface from $\sim 3 \mathrm{~mm}$ (low iota part) to $\sim 10 \mathrm{~mm}$ (high iota tail). The exposure time has been set manually in the range from 1 to $9 \mu \mathrm{s}$ for each discharge, depending on the infrared emission. The Planck law based calibration has been carried out in the laboratory before the campaign including all the actual machine windows and designed optical routes, against a cavity radiator with known emissivity and temperature. The relation between the received digital levels and the temperature has been compiled into look-up tables. The camera control and temperature conversion have been incorporated into a software platform for a real time observation of surface temperature during the experiments [15].

\section{2D Projection method}

Instead of analyzing thermographic data in the distorted view as shown in Figure 3, one could on the other hand, restore the distorted camera data to $2 \mathrm{D}$ planes, for a much easier visualization and accurate comparison with numerical simulations. Thus a 2D projection (Figure 5) of the divertor surface from the 3D CAD model (Figure 4) is produced. Each divertor component is a series of consecutive thermal insulated target elements or the so called divertor fingers, as can be seen in Figure 4. The divertor fingers are flat elementary parts, which reproduce the 3D target shape.

For the preparation of the projection, the plasma facing surfaces (black and blue triangle meshes in Figure 4) of one half-module are extracted from the full CAD geometry. During this process, the original triangles describing the $3 \mathrm{D}$ geometries in the CAD model is maintained. By including the CAD triangles into the projection, one could later render any $2 \mathrm{D}$ analysis on the projection plane back to the 3D geometry via interpolations in barycentric coordinates [24], which will be shown in section 4. After the extraction, the target surfaces in one half module are duplicated ten times due to the identical design of TDUs as described in the introduction.

The 2D projection method developed here is an orthogonal projection of the $3 \mathrm{D}$ divertor surface onto a $2 \mathrm{D}$ plane. There are mainly two applications of this method in this paper:

1. Regards the whole divertor plate as the subject of the method in order to provide a general platform for easy visualization and comparison of 
the overall divertor footprints of temperature or heat flux from different half modules. Since the whole divertor plates in W7-X are slightly curved, such direct projection does not preserve distance nor area. For accurate estimation of power loads, one should use the second application.

2. Apply the method to individual finger for quantitative tasks such as calculating heat flux, integrating powers, etc. The resulted 2D finger coordinate would preserve the accurate geometry, since the finger is designed and manufactured to be flat. The calculated results from all fingers can then be combined and visualized altogether on the developed $2 \mathrm{D}$ target map or the original 3D divertor surfaces.

To project the target surface to a $2 \mathrm{D}$ plane, one needs to define the origin $r_{o}$, the normal vector $\vec{n}$, as well as two orthogonal vectors $\overrightarrow{e_{1}}, \overrightarrow{e_{2}}$ for the axes directions of the plane in the 3D Cartesian coordinate. These vectors are visualized in Figure 4. The origin $r_{o}$ is defined as the weighted average of the center positions of all triangles of the target surface, with the weight being the area of each triangle. Similarly the $\vec{n}$ is the weighted average of normal vectors of all triangles pointing towards magnetic axis, with the weight being the area of each triangle. A temporary vector $\overrightarrow{e_{t}}$ is defined in order to calculate the axes directions: $\overrightarrow{e_{1}}=\overrightarrow{e_{t}} \times \vec{n}$. For vertical target, $\overrightarrow{e_{t}}$ is the unit vector parallel with the $\mathrm{Z}$ axis, with the sign pointing towards the magnetic axis from $r_{o}$. For horizontal target, $\overrightarrow{e_{t}}$ is the unit vector pointing towards the machine center $(0,0,0)$ from $r_{o}$. The other axis direction in the 2D plane can be derived by $\overrightarrow{e_{2}}=\vec{n} \times \overrightarrow{e_{1}}$. One could project and derive the $2 \mathrm{D}$ coordinate $t_{1}$ and $t_{2}$ of any $3 \mathrm{D}$ point $r_{p}$, with a simple equation:

$$
r_{p}=r_{o}+t_{1} \cdot \overrightarrow{e_{1}}+t_{2} \cdot \overrightarrow{e_{2}}+s \cdot \vec{n}
$$

where $s$ is the normal separation (distance) between the plane and the point $r_{p}$. Due to the orthogonality, we get $t_{1}=\overrightarrow{e_{1}} \cdot\left(r_{p}-r_{o}\right), t_{2}=\overrightarrow{e_{2}} \cdot\left(r_{p}-r_{o}\right)$. As the origin, $r_{o}$ has the $2 \mathrm{D}$ coordinate $\left(t_{1}, t_{2}\right)=(0,0)$.

Figure 5 shows the projected targets overlaid with the temperature rise on the carbon surface of one divertor module heated by the plasma within $10 \mathrm{~s}$ (electron cyclotron heating power of $3 \mathrm{MW}$, line integrated electron density of $\left.3.5 \times 10^{19} \mathrm{~m}^{-2}\right)$ measured from the infrared camera during the experiments. Due to the varied distances $(\sim 1.5$ to $\sim 4 \mathrm{~m})$ from different parts of the target to the camera and the optical distortion from the wide-angle optics, the spatial resolution in between two camera pixels varies on the target surfaces. As a result the data points are less dense in the high iota tail and vertical target, as compared with the lower iota part in the horizontal target. The 


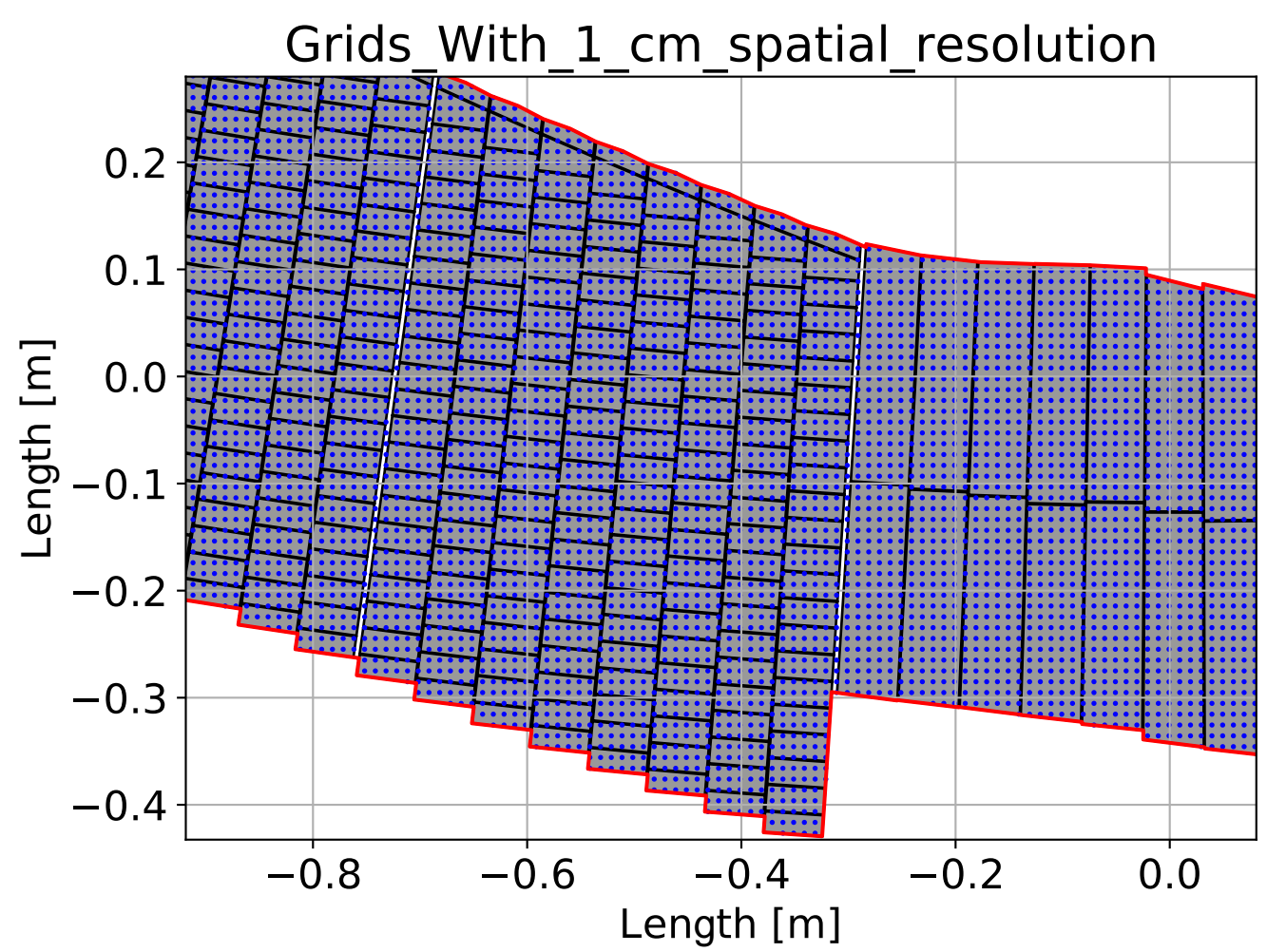

Figure 6: Grid defined with $1 \mathrm{~cm}$ spatial resolution inside the projected surface.

good mapping quality is confirmed by the clear separation of the leading edges in between fingers. Langmuir probe arrays are installed on two fingers of the horizontal target. Their tips are standing out of the surface and are visible from the infrared emission. Certain boundary areas on the horizontal target are not visible from the view of the camera, because the line of sights are blocked by other in-vessel components in front of the targets, e.g. the green area marked in the figure is blocked by the outer baffle plate, which can be seen in Figure 3.

\section{Grid interpolations}

The 2D projections of the target surfaces described in section 3 for all ten divertor units are identical, due to the machine symmetry. Nevertheless the optical route of the immersion tube for each half module is not the same, which results in a different distribution of the projected camera pixels on the $2 \mathrm{D}$ plane. In order to compare the temperature distributions among half 


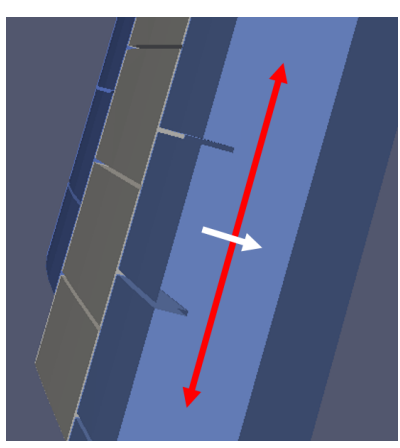

(a)

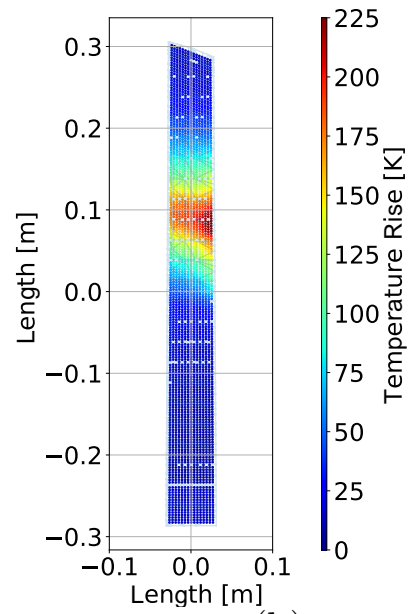

(b)

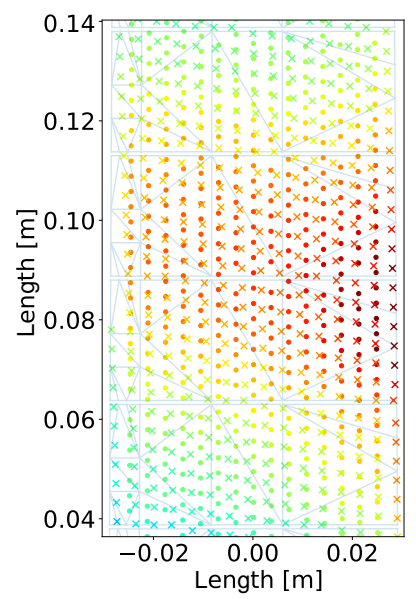

(c)

Figure 7: (a) Cross section of a divertor finger, two heat diffusion direction: perpendicular to the surface (white arrow) and parallel with the longer side of the finger (red arrow). (b) The $2 \mathrm{D}$ projection of a finger with interpolated lines prepared for heat flux analysis. (c) A zoom in figure from (b) showing the scattered camera pixels (cross) and the interpolated points (dots).

modules in a quantitative manner, a universal grid need to be interpolated inside the divertor surface from the scattered camera data.

A simple idea is to create and interpolate a $2 \mathrm{D}$ grid, which covers the whole projected divertor surface region. An example is shown in Figure 6. In this way the temperature data from ten divertor units can be compared directly at each individual grid point at identical position. The grid points created with this method are homogeneously distributed within the boundary of the whole divertor surface, including the gaps in between the fingers.

This simple grid is often used in a fast evaluation of the target symmetry using the interpolated map of the temperature rise during the inter-discharge period, when an immediate decision has to be made for the next discharge. However for post-analysis requiring accuracy, this method is not a good option because of two drawbacks:

1. As discussed in section 3 , the $2 \mathrm{D}$ projection of the whole target is not distance or area preserving. The homogeneous grid points created on this map do not describe the actual geometry of the target, which causes inaccuracy of any estimations using these points.

2. The creation of the grid does not consider the geometry of the divertor 


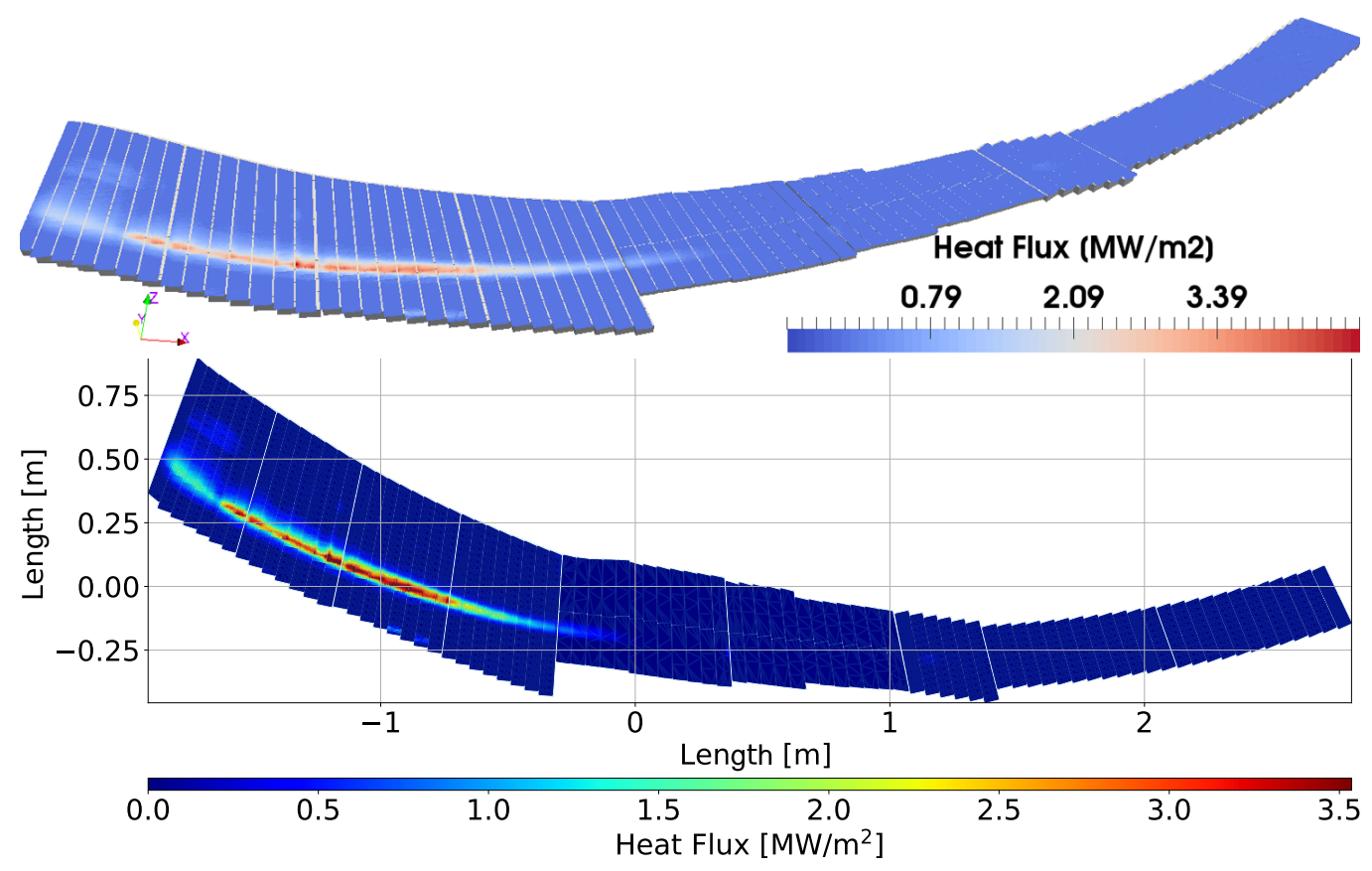

Figure 8: The calculated experimental heat fluxes averaged from all the available lower horizontal targets in a typical low iota magnetic configuration, rendered to the 3D Cartesian coordinate (top) and projected to the surface coordinate (bottom). 
fingers. Thus, it is not useful for the study of leading edges of the fingers. Furthermore, because there is no thermal conduction in between the fingers, this method is not applicable for heat flux calculations.

Thus a more accurate grid model is created, which applies the geometry of each divertor finger separately as the boundary condition.

2D heat diffusion is considered in a finger, i.e with the direction of poloidal and into the finger as shown in Figure 7 (a). The fingers are castellated at the surface with shallow segments of $\sim 4.4 \mathrm{~mm}$ deep and $\sim 0.8 \mathrm{~mm}$ wide in between tiles $(50 \times 25 \mathrm{~mm})$, while the whole finger with total depth of $\sim 28.8 \mathrm{~mm}$ is connected underneath. The toroidal heat diffusion within a finger is estimated to be small, given a rather homogeneous footprint of the strike line in finite toroidal range, limited by the width of the finger $(50 \mathrm{~mm})$. There is no conductive heat diffusion across the fingers, because of the gap in between them. Validations and discussions about the applicability of the $2 \mathrm{D}$ model to the 3D finger can be found in appendix.

Figure 7 (b) shows one of the 2D projection of a divertor finger, rendered with the experimental temperature data. The $2 \mathrm{D}$ projection method is the same as described in section 3, except for the change of the subject from the whole divertor surface (target coordinate) to individual finger surface (finger coordinate). This finger coordinate is essential for an accurate heat flux calculation, because it provides actual dimension of the finger as the boundary conditions. The defined grid points have a spatial resolution of $3 \mathrm{~mm}$ and are distributed along the lines parallel to the longer side of the finger, while the original camera pixels projected to the $2 \mathrm{D}$ plane are scattered as shown in Figure 7 (c).

The interpolation method has the following steps:

1. Delaunay triangulation [25] of all the irregularly distributed camera pixels within the finger.

2. Identification of the vertices of the triangle enclosing each defined grid point.

3. Calculation of the barycentric coordinates [24] for all the defined grid points.

4. Interpolation of the grid points using the weights from the corresponding barycentric coordinates, and the values of the temperature of the registered vertices.

The first three steps are processed once and saved in order to reduce the computation time, given the fact that the cameras are not moving with respect to the machine throughout the experiment. 


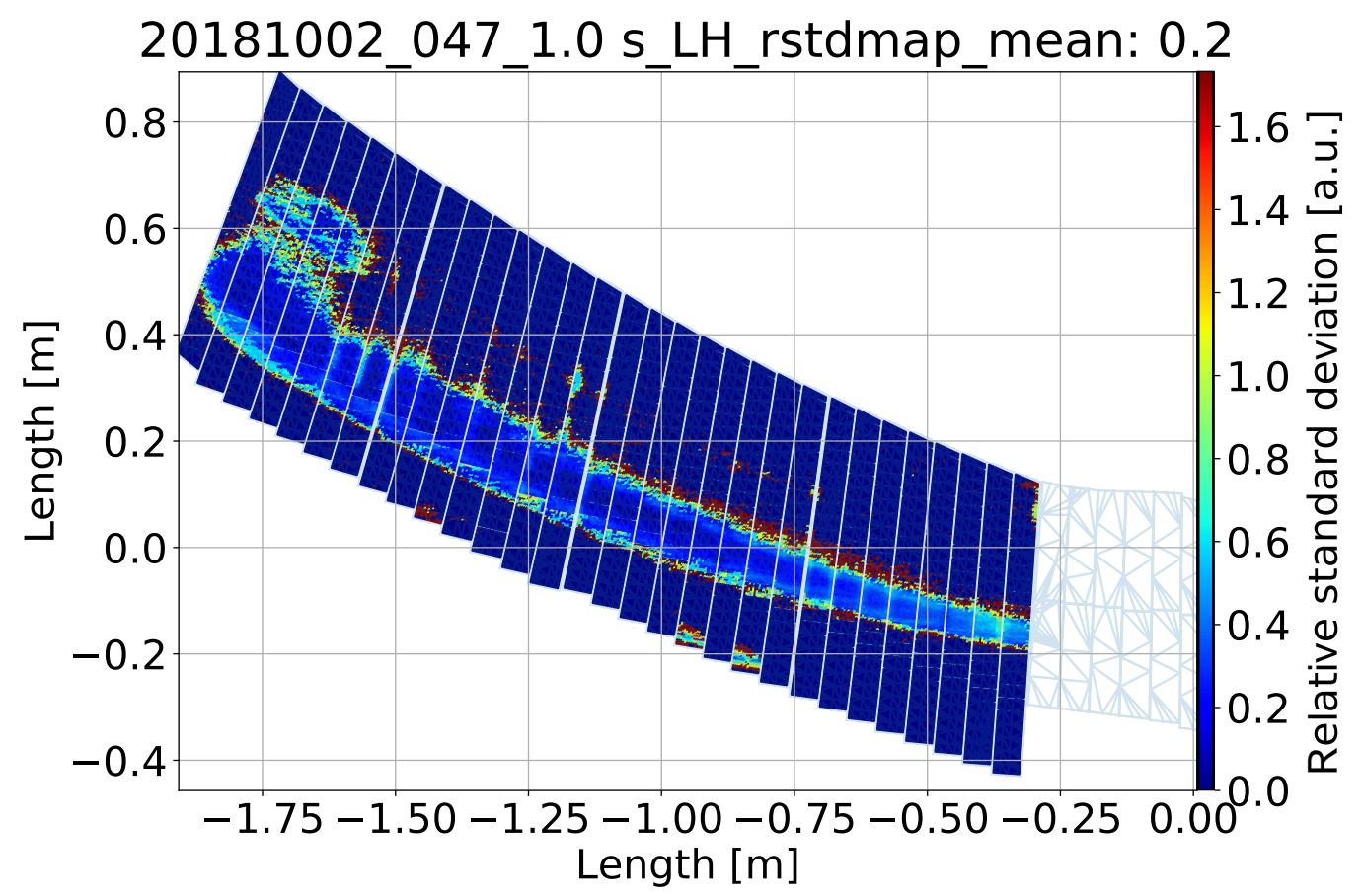

Figure 9: The map of relative standard deviation in the region of lower iota part of the target, calculated using the same data as in Figure 8. The mean value of the rstd map is 0.2 , as a quantity for symmetry estimation.

The equation for the calculation of barycentric coordinates $\left(w_{1}, w_{2}, w_{3}\right)$ of a defined grid point $P\left(t_{1}, t_{2}\right)$ is:

$$
\left(\begin{array}{ccc}
x_{1} & x_{2} & x_{3} \\
y_{1} & y_{2} & y_{3} \\
1 & 1 & 1
\end{array}\right)\left(\begin{array}{l}
w_{1} \\
w_{2} \\
w_{3}
\end{array}\right)=\left(\begin{array}{c}
t_{1} \\
t_{2} \\
1
\end{array}\right)
$$

Here $\left(x_{1}, y_{1}\right),\left(x_{2}, y_{2}\right)$ and $\left(x_{3}, y_{3}\right)$ are the $2 \mathrm{D}$ coordinates of the three vertices. The calculated barycentric coordinates are used to interpolate the value of the grid point $v_{p}$ from the values of the vertices $v_{1}, v_{2}, v_{3}$.

$$
v_{p}=w_{1} \cdot v_{1}+w_{2} \cdot v_{2}+w_{3} \cdot v_{3}
$$

2D heat diffusion calculations [8] are performed on the interpolated lines of temperature to derive the heat flux to the surface, with the advantage that the actual length of the finger is used, which is not affected by the viewing angle or optical distortions. For visualizing the heat flux distributions on 


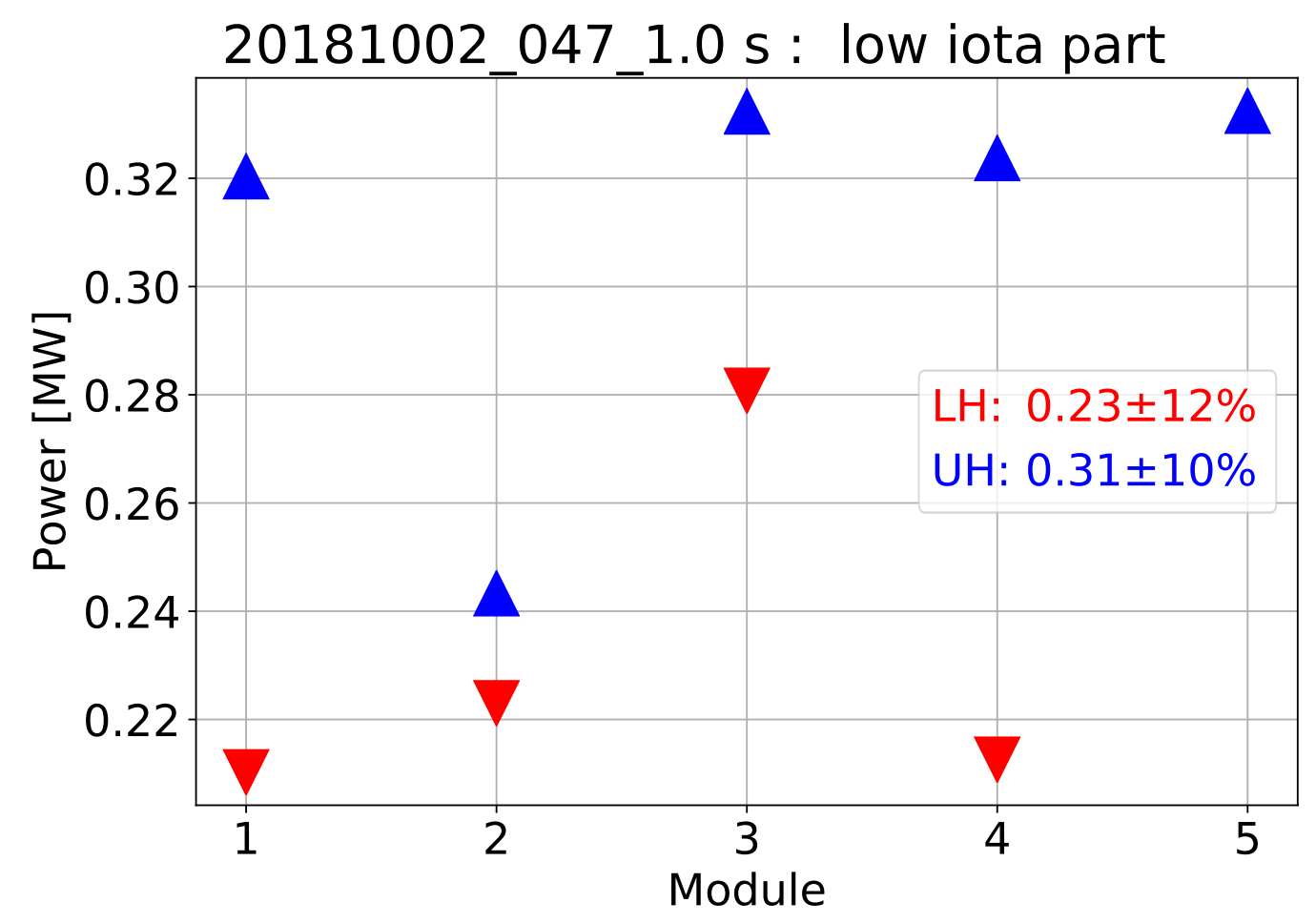

Figure 10: Integral power loads to the horizontal targets in the region of low iota part. The mean and rstd value are labelled in the legend for lower horizontal (in red) and upper horizontal targets (in blue) separately. 
the whole target, the defined lines on all fingers (typically 13 - 15 lines for one finger) with calculated heat flux could be projected to either the 3D Cartesian coordinates or the 2D plane of the whole target surface as defined in section 3. In order to render the heat flux data to $3 \mathrm{D}$, for each grid point on the defined lines in the finger projection, a barycentric coordinate inside the background triangle (as shown in Figure 7 (c)) projected from the 3D CAD model is calculated. Together with the known locations of the vertices in the 3D and the target coordinates, the visualization of the grid points of all fingers can be obtained, as shown in Figure 8.

In this example (discharge number 20181002_047 at $1 \mathrm{~s}$ ), the heat flux data on each grid point is an average over four lower horizontal targets (the endoscope camera monitoring module five lower divertor was not available for this discharge). Such average is performed separately for the lower and upper targets, because experimentally a systematic displacement of the strike-line locations between lower and upper targets is found. This is considered mainly due to the particle drifts effect, since this displacement has been found to be opposite in reversed field experiment under otherwise same conditions. Detailed explanations for this aspect is not covered in this method paper.

Figure 9 shows one capability of the methods, where a map of relative standard deviation (rstd, defined as the population standard deviation divided by the mean value) is produced alongside the averaged heat flux map shown in Figure 8, to support the quantification for the level of target symmetry of power loads. In this discharge under low iota configuration, only the low iota part on the target is calculated for the rstd map. An average of the rstd map in this region results in a dimensionless value of 0.2 , which could be used as a quantity to compare with other experimental discharges aiming for better symmetry. Integral power loads can also be calculated and compared quantitatively for all the targets, using the described area-preserving finger mappings. In Figure 10 the power loads are integrated in the region of low iota part for all the horizontal targets. The general up-down asymmetry of the power loads is considered to be caused again by the particle drifts, while more power loads have been observed on the lower targets in a comparative experiment with magnetic field reversed compared with this example. Deviations of the power deposition in module 2 upper and module 3 lower targets with respect to other upper or lower targets are considered to be caused by finite divertor misalignments and error fields. More researches with respect to this issue are ongoing. 


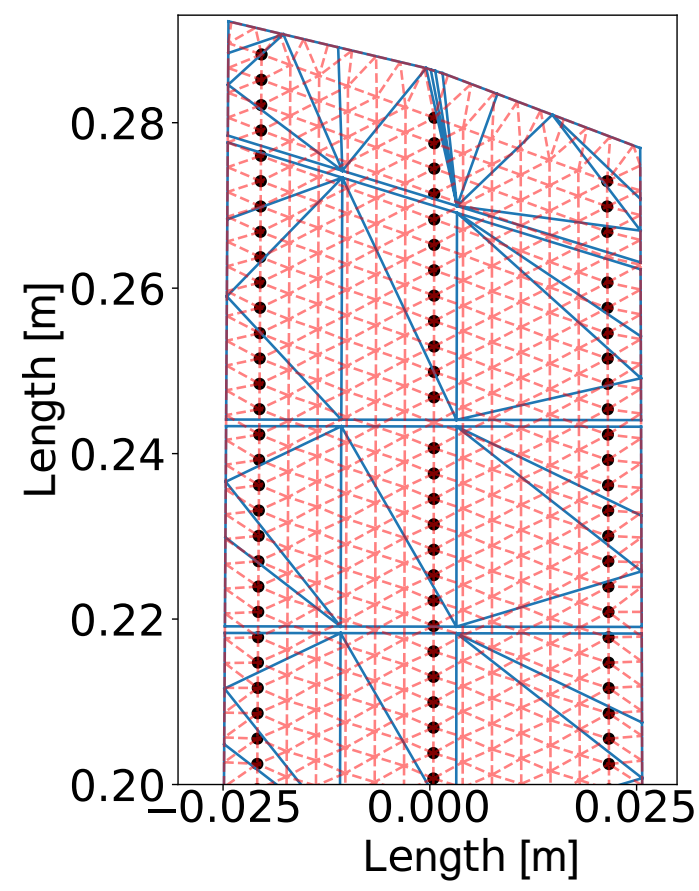

Figure 11: The triangle mesh subdivided by Delaunay triangulation projected to the finger plane. The blue solid lines are the original CAD representation of the surface. The red dashed lines are the results from the Delaunay triangulation. Black dots are selected as the very left, the middle and the very right lines among the lines defined in Figure 7. 


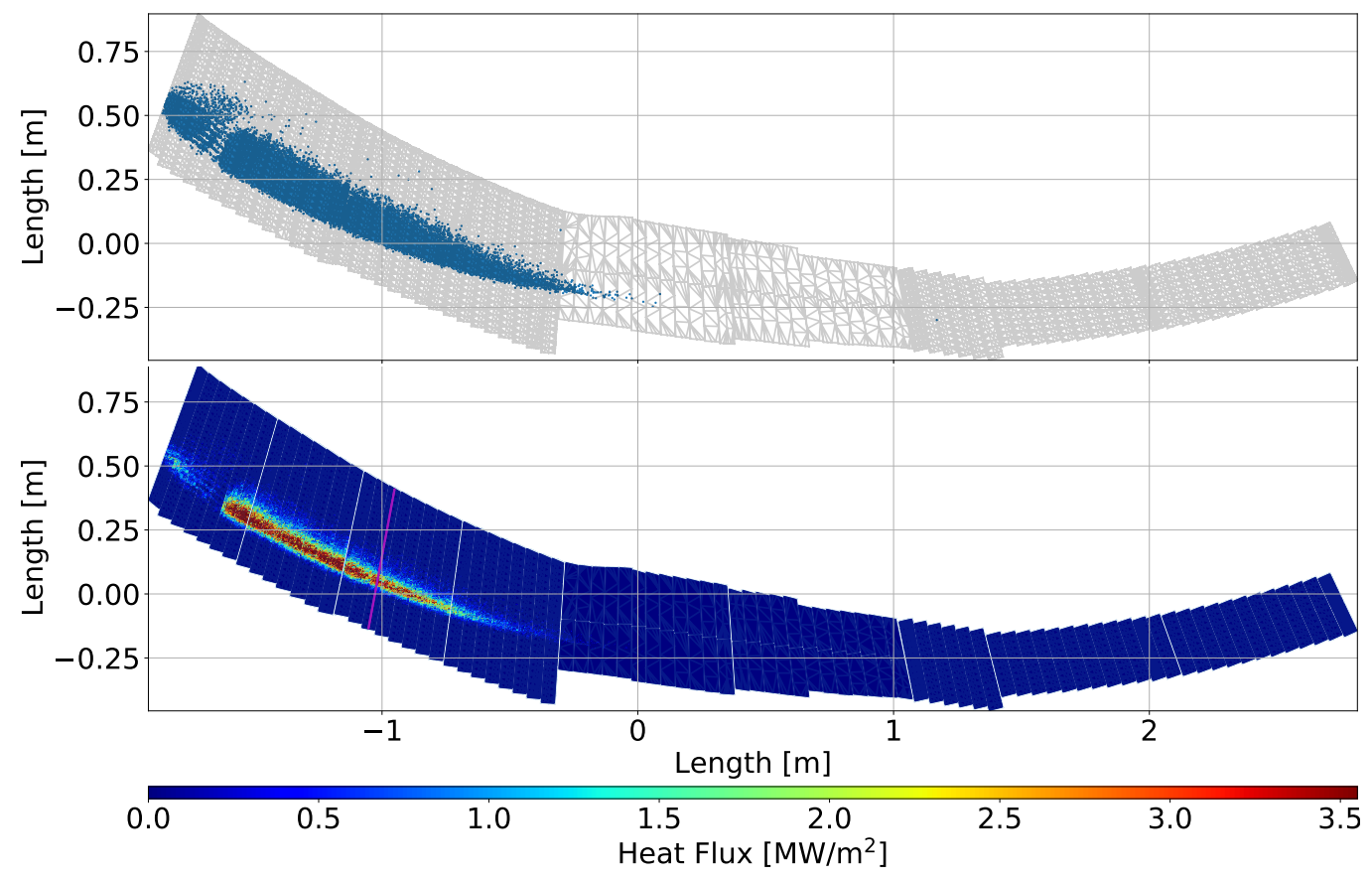

Figure 12: Diffusive field line tracing simulation on the horizontal target in low iota magnetic configuration. The top figure presents the intersection points with the original CAD triangles as background. The heat fluxes are estimated in the bottom figure with arbitrary scale to fit the experiments. The middle line of the finger used in Figure 13 is labeled in magenta. 


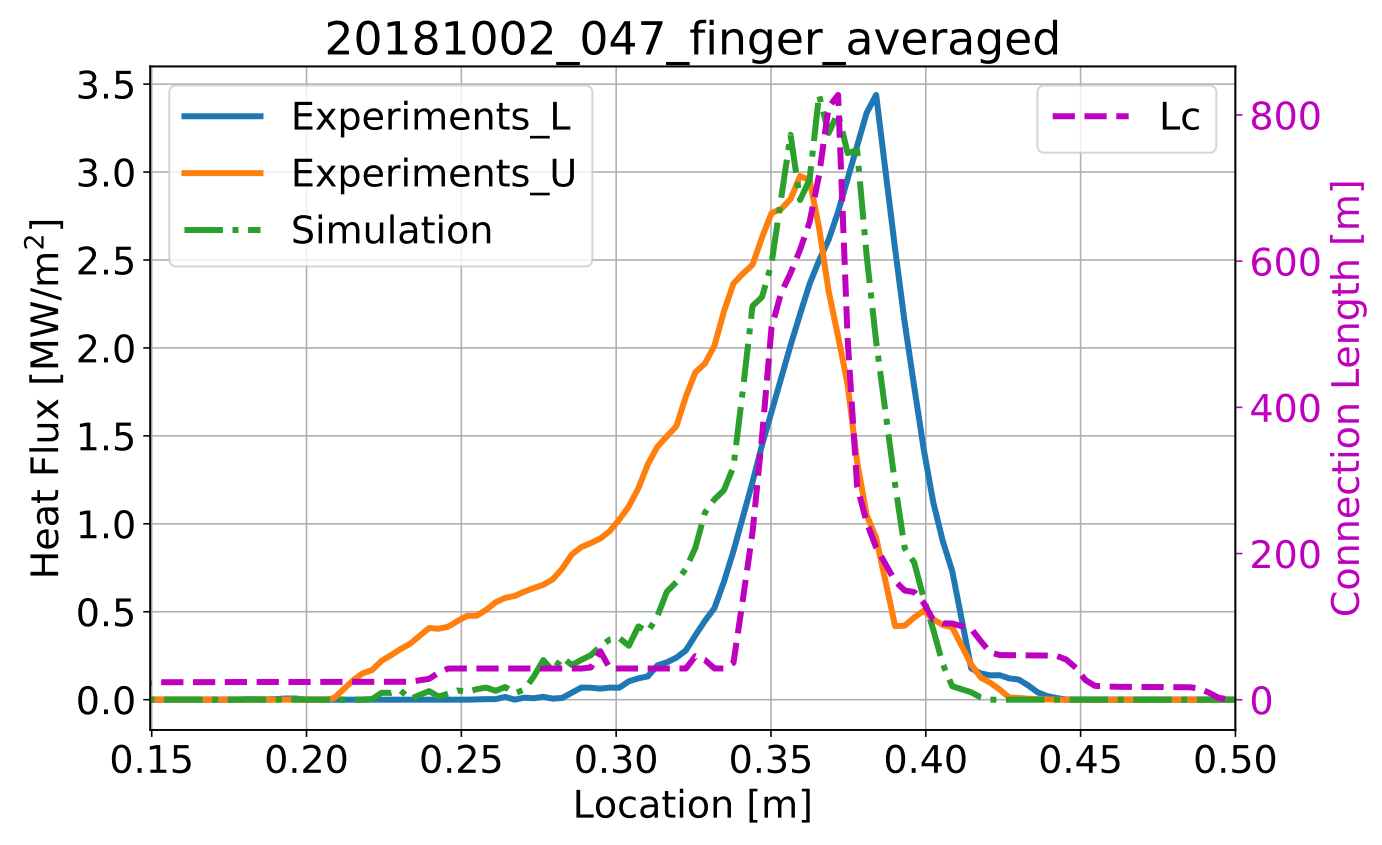

Figure 13: The comparison of the simulated heat flux profile (green), target to target connection length profile (magenta), the experimental heat flux profile on the lower targets (blue) and on the upper targets (orange). The location zero is defined at the pumping gap end of the finger (see Figure 2). The amplitude of the simulated heat flux is arbitrary and scaled to the experimental values. 


\section{Divertor subdivision for simulations}

For a better understanding of the divertor physics, a quantitative comparison between experimental results and simulations is important. For the comparison of divertor footprints in 3D boundary like in W7-X, it is ideal to apply the identical mapping for both modelling and measured results. The well established diffusive field line tracer [7] has the possibility to register the 3D coordinates of the intersection points on the plasma facing components of the test particles, which are simulated and traced from the confined region. An approximate heat flux value (HF) could be estimated by the number of the intersection points $\left(N_{A}\right)$ within certain areas $(\mathrm{A}): H F=N_{A} / N / A \cdot P$, where $\mathrm{N}$ is the total number of the test particles being traced, $P$ is the assumed power reaching the targets.

However the existing CAD model has a nonuniform density of the triangles, i.e. more triangles found in the edge or larger curvature region, while a flat surface has large and only a few triangles. For a detailed comparison with thermography data on the divertor target, which has a minimum spatial resolution of $\sim 3 \mathrm{~mm}$ at the lower iota part viewing from the infrared cameras, such representation from CAD is not sufficient. A subdivision of the divertor target is needed to maintain the quality from simulations.

For an accurate comparison with the experimental data, such subdivision of the triangles as well as the associated projection and counting of the intersection points are done in the identical area-preserving finger coordinate used in the experimental heat flux calculation. Figure 11 shows the comparison between the original CAD model and a subdivision derived from a Delaunay triangulation for a typical finger projected to its own 2D plane. The grid points along the lines defined in section 4 and a set of exterior points of the finger are used as the vertices for the triangulations. With this approach, a mesh with rather homogeneous distribution of the triangles (edge length of $\sim 3 \mathrm{~mm}$ ) can be obtained for each finger. By counting the numbers of intersection points in each triangle, the heat flux of the triangle area can be estimated, and represented by the centroid of the triangle. Finally with the same barycentric interpolation method as described in section 4 but with the vertices being the centroids of the subdivided triangles, the heat fluxes on the identical grid points as experimental results can be achieved.

This subdivided divertor mesh is developed as a universal interface, which can be coupled to other code. The simulated intersection points from the more advanced Monte Carlo method like EMC3-EIRENE [18] could also be mapped to this surface model for a direct comparison with experimental results.

As an example shown in Figure 12, the heat flux distribution on the 
horizontal target in a low iota magnetic configuration is simulated and can be visualized and compared with experimental results in Figure 8 directly. In Figure 12, 88473 test particles are intersected by the horizontal target for a good statistics, and the density of them are reflected as the heat flux. The strike line represented by the intersection points looks more broader than the heat flux. This is because the most dense area only concentrates at the peak of the strike line, although a few test particles could diffuse to a remote area surrounds the peak resulting in much lower heat flux.

For a quantitative comparison, one should apply the analysis on the finger coordinate, e.g. for an accurate characterization of the strike line with respect to the strike-line width and locations, as shown in Figure 13. To reduce the noise from the bolometric infrared detector, an average over all the line profiles within the finger is applied for the experimental results. This averaging is also favoured to extract the main characteristic of the strike line, instead of the possible influence from local hot spot or specific feature in individual heat flux profile. Same line averaging process has to be applied also for the simulated results to make a valid comparison with experiments for magnetic topology studies. In this example, the profiles for each target are averaged from all the thirteen lines defined within this specific finger by a scaling and interpolation process with respect to the middle line. Firstly the location coordinates of all the lines are linearly scaled to the length of the middle line within this finger:

$$
S_{\mathrm{i} \_ \text {scaled }}(x)=S_{\mathrm{i}}(x) \cdot\left(L_{\text {middle }} / L_{\mathrm{i}}\right)
$$

where $L_{\mathrm{i}}$ is the total length of an individual line, $L_{\text {middle }}$ the total length of the middle line, $S_{\mathrm{i}}(x)$ is the original location coordinate of the individual line. Then an 1D linear interpolation is applied for each profile with respect to the location coordinate of the central line $\left(S_{\text {middle }}(x)\right)$. This averaging method is robust and reasonable for the W7-X finger geometry, even though it would as well smooth and broaden the heat flux profile slightly as any other averaging methods. However as long as the identical approach is applied for both experimental and simulated data, the results are comparable.

In this example, the experimental results are also averaged over all the five upper and five lower targets respectively. The simulated heat flux and the connection length peak at the same location, while the experimental strikeline locations are slightly shifted. The differences in the strike-line width and the position between the upper and lower targets are considered to be influenced by the particle drifts, which are out of the scope of this paper. 


\section{Summary}

In this paper, methods developed for quantitative studies of the divertor heat loads on W7-X are presented. The full coverage of the divertor modules by the ten wide-angle thermographic systems for operation safety, potentially provides key measurements for a better understanding of the 3D effects on the heat transport. The 2D projection method creates a standard mapping for visualizing and comparing the thermography analysis. The finger projection and line interpolation as a prerequisite enables the accurate heat flux calculations from the recorded surface temperature evolution. With interpolations from barycentric coordinate, for each grid point defined in a finger coordinate, a 2D surface coordinate and a 3D coordinate are also registered for visualization with different purposes. In order to preserve the spatial resolution from the infrared diagnostics (up to $\sim 3 \mathrm{~mm}$ ), subdivided meshes are created by Delaunay triangulation for heat diffusive field line tracing. The simulation and experimental results can thus be compared quantitatively in identical coordinate system. The methods introduced in this paper will and have been applied in various engineering and scientific tasks.

\section{Acknowledgement}

I thank Jiawu Zhu for the ANSYS ${ }^{\circledR}$ calculations, Michael Endler for the parameters of leading edges, Michael Rack for the guidance of the field line tracer and Joachim Geiger for the helpful discussions and explanations of magnetic configurations in W7-X.

This work has been carried out within the framework of the EUROfusion Consortium and has received funding from the Euratom research and training programme 2014-2018 and 2019-2020 under grant agreement No 633053. The views and opinions expressed herein do not necessarily reflect those of the European Commission.

\section{Appendix. Validation of 2D heat flux calcula- tion on TDU divertor element}

For calculating the heat flux profile on the TDU divertor element, a 2D heat diffusion equation is solved using a numerical discretization of forwardtime central-space method [8] (so called 2D THEODOR code), with the 


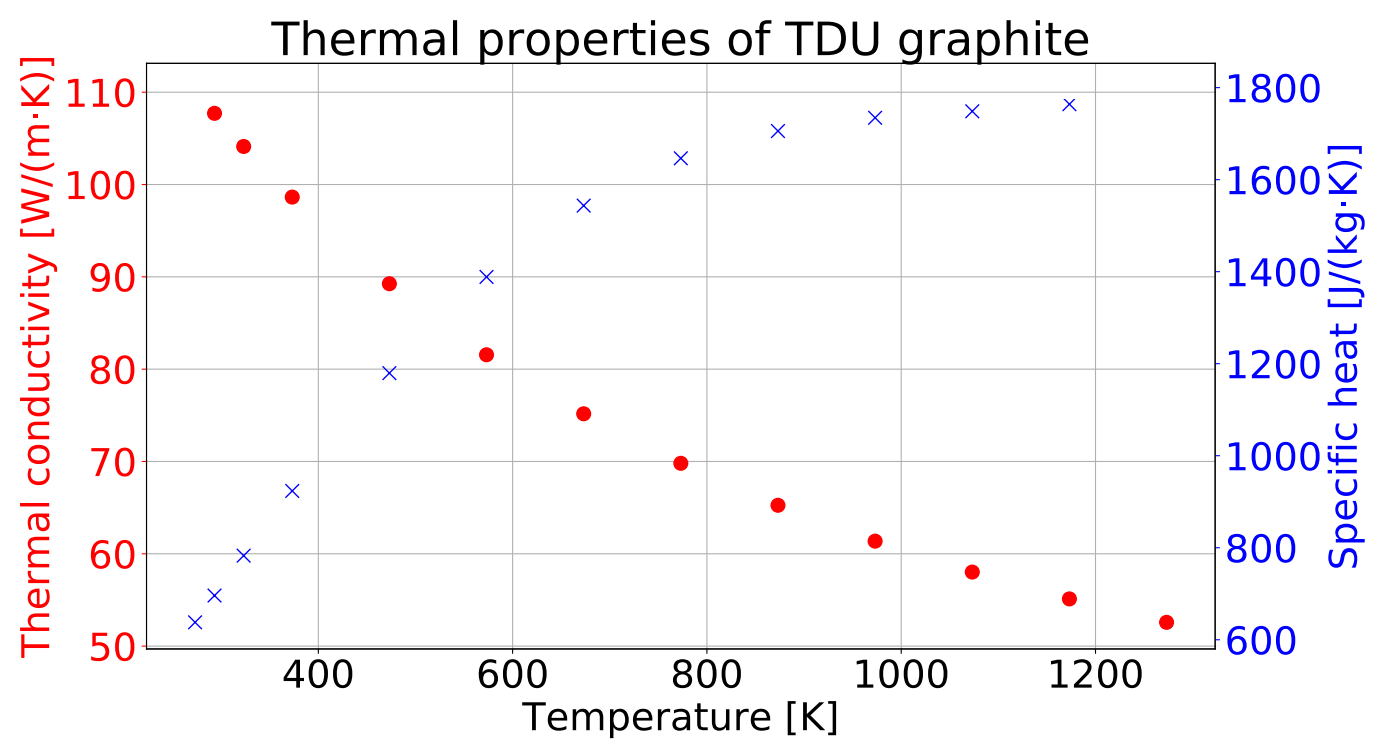

Figure A.1: The thermal conductivity (red) and specific heat (blue) of the TDU graphite.

interpolated 1D temperature evolution:

$$
\rho c_{p} \frac{\partial T}{\partial t}=\nabla(\kappa \nabla T)
$$

where $\rho$ is the volumetric mass density, $\kappa$ the heat conductivity, and $c_{p}$ the specific heat capacity of the fine graphite used for the TDU. Figure A.1 shows the thermal properties of the graphite, which is isotropic and temperature dependent.

The model in this code assumes a continuous $2 \mathrm{D}$ slab with no heat diffusion in the toroidal direction as described in Figure 7 (a), which is imperfect for the 3D finger because of the designed castellation structures and the observed toroidal asymmetry caused by leading edges. Therefore, validations against a full 3D model with finite element calculations from ANSYS $^{\circledR}$ are presented here as well as discussions for the applicability of this 2D model in the 3D finger. A Gaussian function is applied as the input heat flux profiles for ANSYS ${ }^{\circledR}$ :

$$
P_{\text {surf }}=A \cdot \exp \left(\frac{-(x-\mu)^{2}}{2 \sigma^{2}}\right)
$$

where the peak of the heat flux is set as $A=3.5 \mathrm{MW} \mathrm{m}^{-2}$ with the location of the peak at the center of a tile, and the width of the profile is controlled by $2 \sigma=50 \mathrm{~mm}$. The initial equilibrium temperature in the finger is $295.15 \mathrm{~K}$, with no thermal radiation considered. This Gaussian like heat flux is loaded 
Toroidal symmetry case: temperature distribution $(\mathrm{K})$ at $10 \mathrm{~s}$

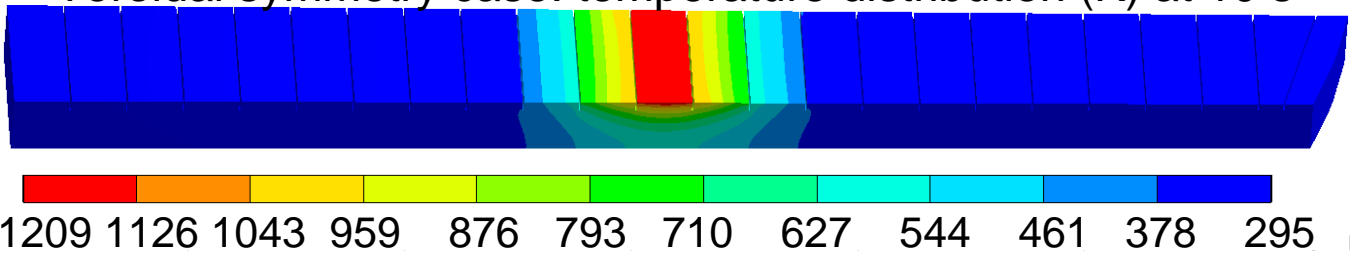

Figure A.2: The temperature distribution resulted from ANSYS ${ }^{\circledR}$ analysis in a divertor finger after $10 \mathrm{~s}$ Gaussian like toroidally symmetric heat load applied at tile center.

onto the divertor finger for $10 \mathrm{~s}$ constantly. To reduce the calculation time, the time step is set to $\Delta t=30 \mathrm{~ms}$, which fulfills the stability criterion for the explicit scheme used in the 2D model:

$$
\frac{D \Delta t}{\Delta x^{2}} \leq 0.5
$$

where the spatial resolution $\Delta x^{2}$ used for the finite element analysis is $\sim$ $2.5 \mathrm{~mm}$, heat diffusivity $D=\frac{\kappa}{\rho c_{p}}$. The resulting temperature response from ANSYS ${ }^{\circledR}$ is then used as the input of the 2D THEODOR code for heat flux calculation. Finally, a comparison between the inversely calculated heat flux from the 2D THEODOR code and the original input heat flux for the 3D ANSYS ${ }^{\circledR}$ analysis can be made to estimate the deviations caused by the limitations of the 2D model.

Two cases are simulated to estimate following effects:

1. Thermal barriers caused by castellations.

A toroidally symmetric heat flux within the finger is assumed, with the above described Gaussian function in radial direction. The thermal response after the $10 \mathrm{~s}$ heat load can be seen in Figure A.2. The heat flux evolution is inversely calculated by the 2D THEODOR code from the ANSYS ${ }^{\circledR}$ temperature output, as shown in Figure A.3. Figure A.4 suggests a finite deviations caused by the castellations between the calculated heat flux and the original input. At each tile edge, a clear step in the calculated heat flux profiles can be seen, which is due to the radial temperature step of ANSYS ${ }^{\circledR}$ output. The profiles are gradually broadened over time and the peak heat flux drop from 3.5 to $3.25 \mathrm{MW} \mathrm{m}^{-2}$. By integrating the heat flux profile along the length $\left(\mathrm{MW} \mathrm{m}^{-1}\right)$, a deviation up to $10 \%$ is found between the integral calculated and input heat flux, which is considered to be acceptable. 


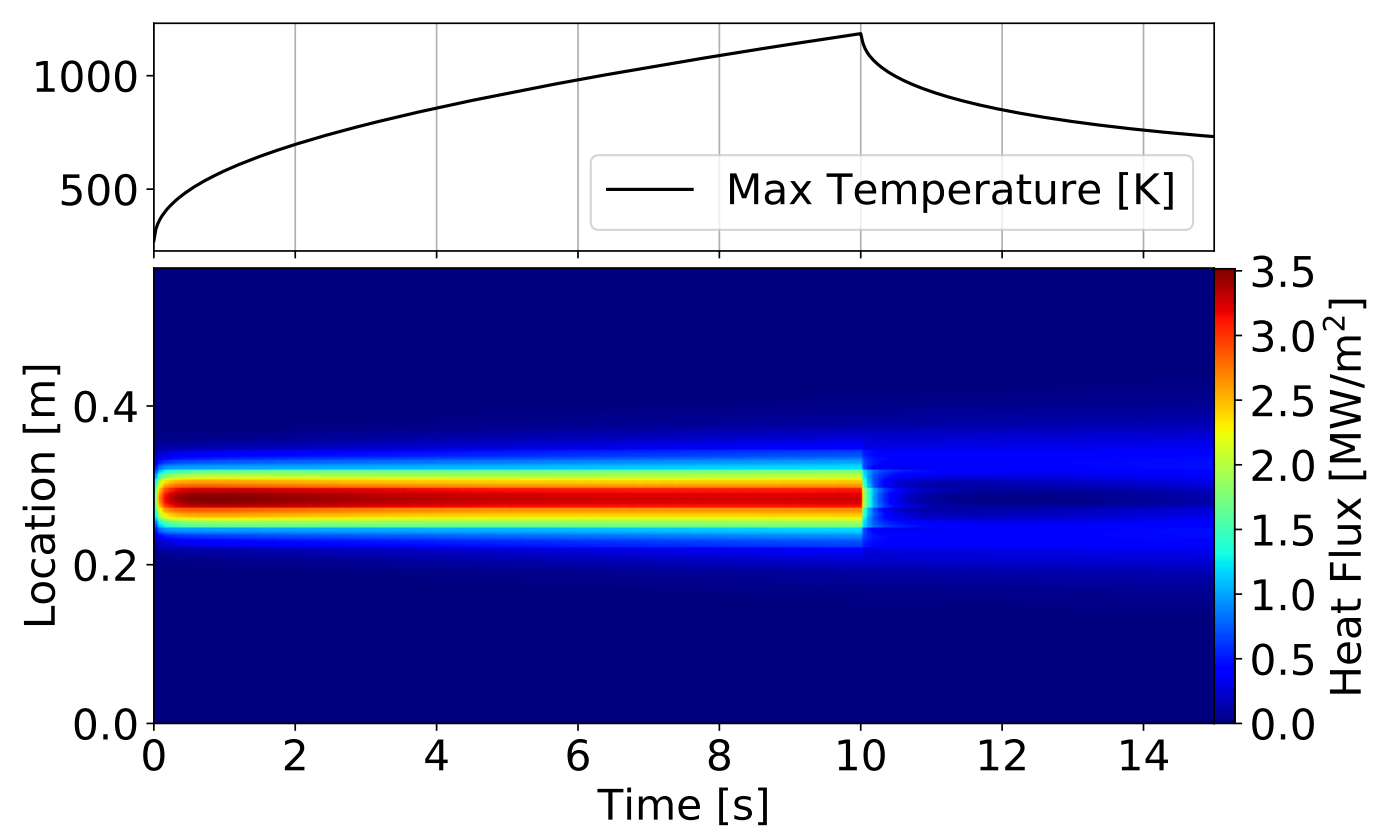

Figure A.3: The maximum temperature from ANSYS ${ }^{\circledR}$ (top) and inversely calculated heat flux evolution from THEODOR (bottom) in toroidal symmetric case.

2. Toroidal asymmetry due to leading edge.

Toroidally asymmetric temperature within a finger has been predicted at the leading edges [21] and observed in the experiments. In addition to the toroidally symmetric $P_{\text {surf }}$ applied in case 1 , a heat flux perpendicular to the side of the finger is designed:

$$
P_{\text {side }}=P_{\|} \sin \gamma=P_{\text {surf }} \frac{\sin \gamma}{\sin \alpha}
$$

where typical values in W7-X are used for the glancing angle of the magnetic field lines onto the top surface $\alpha=3^{\circ}$, and to the side surface $\gamma=75^{\circ}$. As a result, $P_{\text {side }}$ is also a Gaussian like profile radially, but with the calculated peak of $65 \mathrm{MW} \mathrm{m}^{-2}$. For a schematic view and the definition of the angles, one can refer to Figure 4 in ref. [21]. We apply $P_{\text {side }}$ to a very deep depth of $h_{\mathrm{LE}}=0.5 \mathrm{~mm}$, which is referring to one of the largest height measured before and after OP1.2a of the leading edges in between fingers. Thus, it demonstrates rather an extreme case possible in the experiments for the toroidally varying heat distribution caused by the leading edge. Figure A.5 shows the thermal response in the finger for the designed heat loads after $10 \mathrm{~s}$. The resulted temperature distribution is similar to typical experimental observations on the 


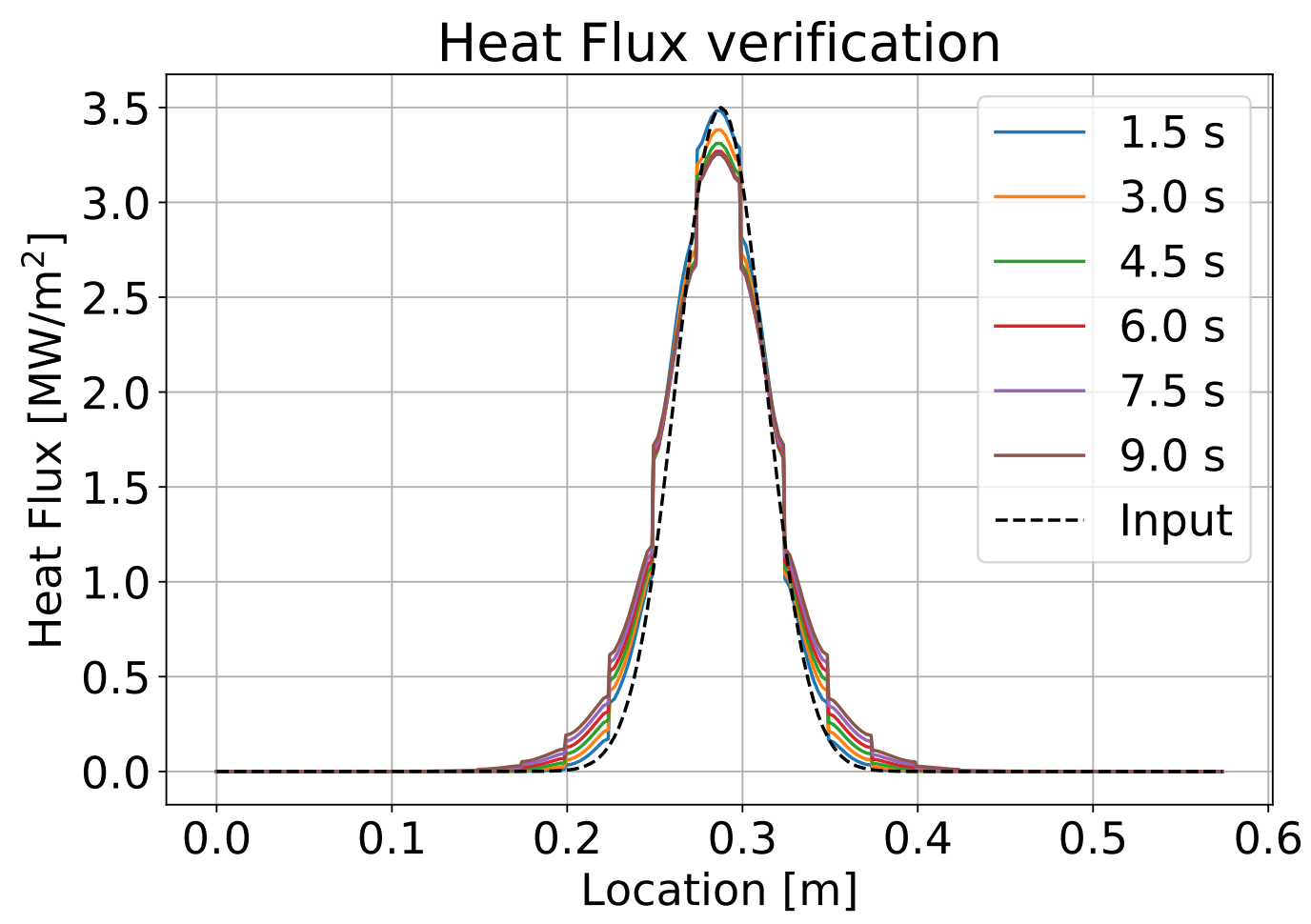

Figure A.4: Comparisons between the calculated heat flux profiles at different time and the input heat flux in toroidal symmetric case.

Leading edge case: temperature distribution $(\mathrm{K})$ at $10 \mathrm{~s}$

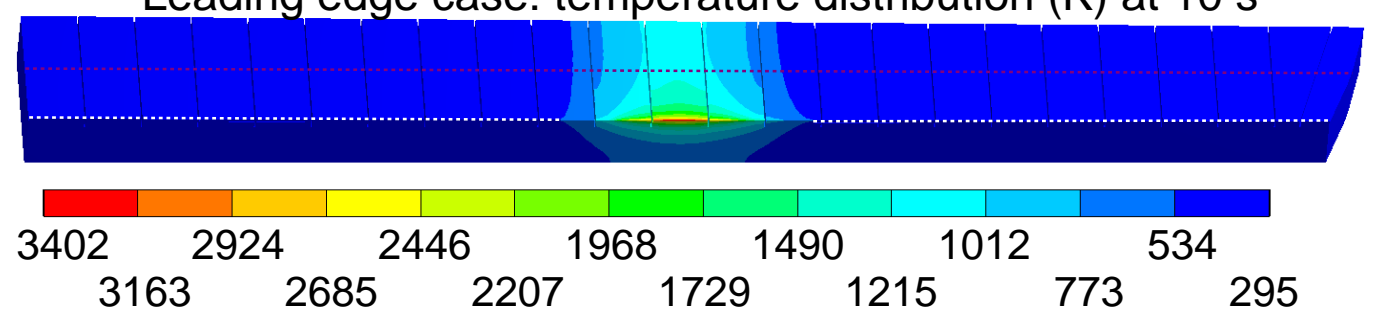

Figure A.5: The temperature distribution in a divertor finger at $10 \mathrm{~s}$, under a constant Gaussian like toroidally symmetric heat load from the top (case 1) and a leading edge heat load from one side. The dashed lines indicate the hottest line (white) and the central line (purple) of the finger for the analysis in Figure A.6 and Figure A.7 respectively. 


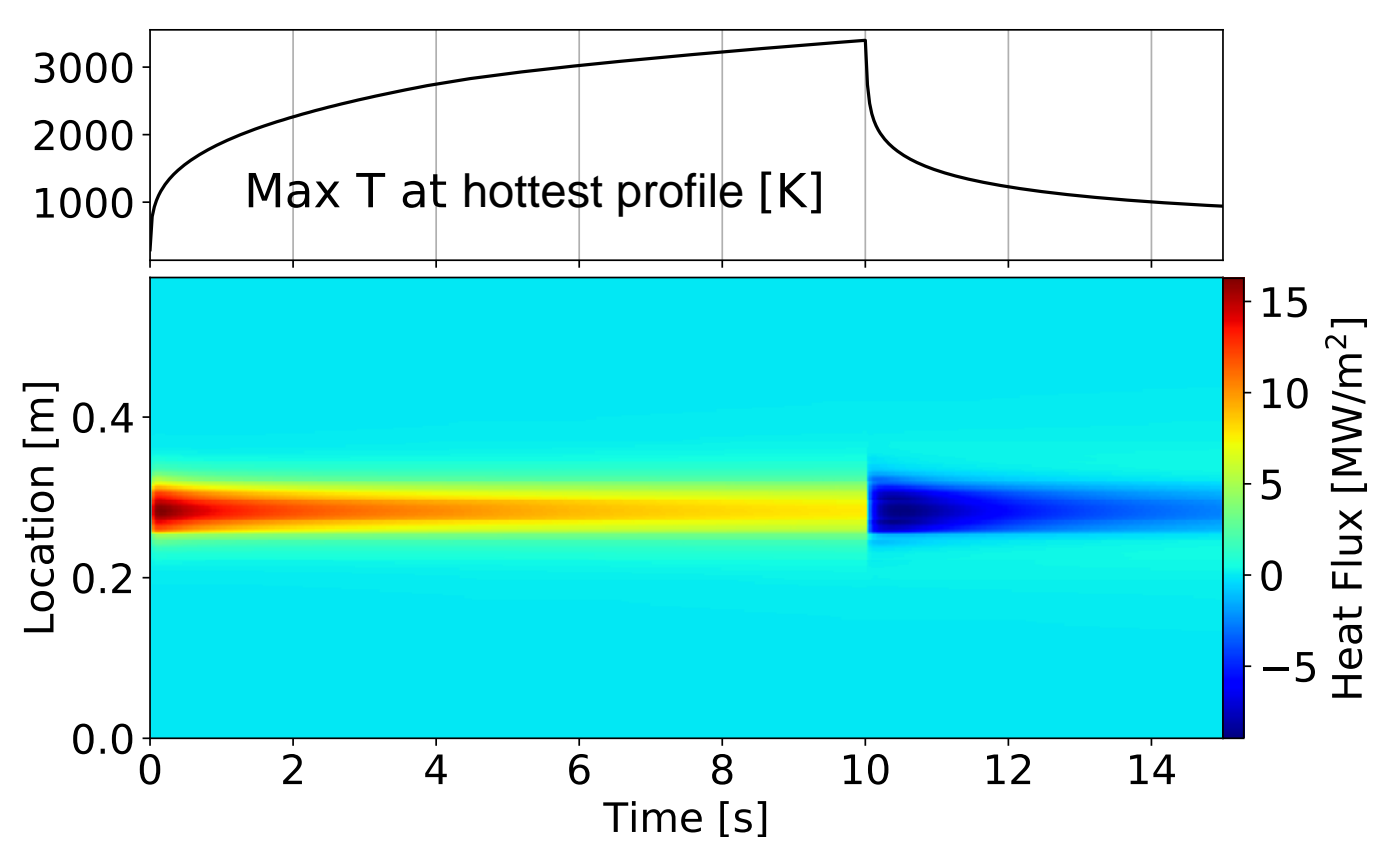

Figure A.6: The maximum temperature and inversely calculated heat flux evolution at the hottest line of the finger for the leading edge case.

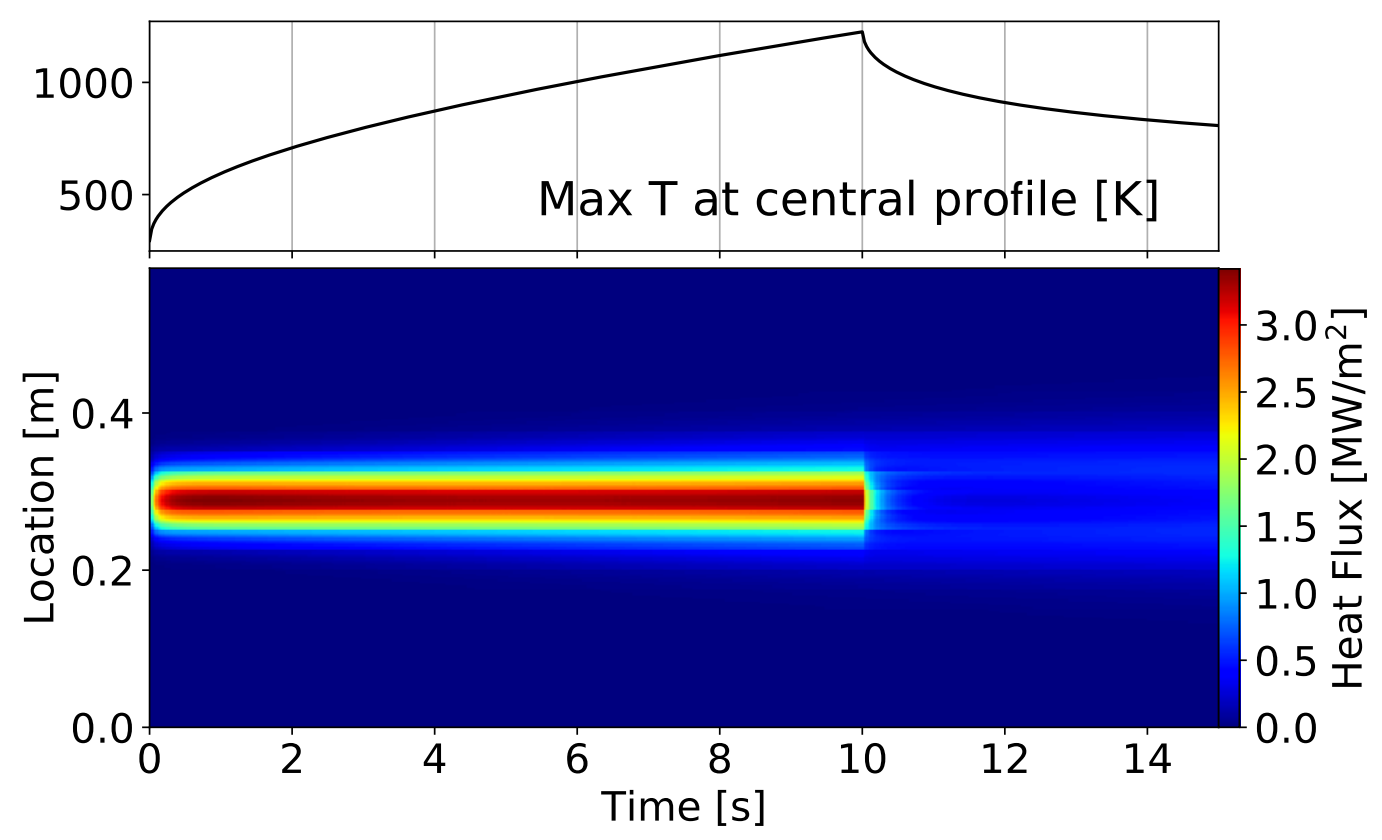

Figure A.7: The maximum temperature and inversely calculated heat flux evolution at the central line of the finger for the leading edge case. 

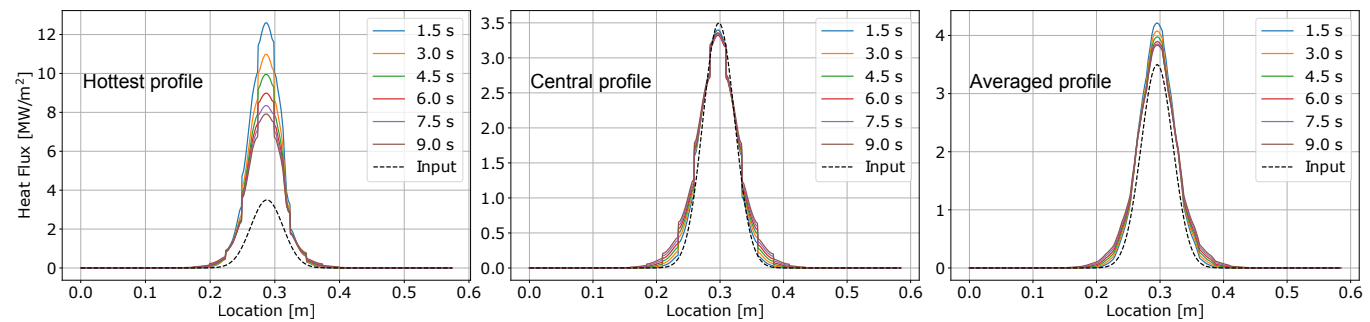

Figure A.8: Comparisons of the calculated heat flux profiles for the hottest line, the central line and the line averaged from all profiles in this finger for the leading edge case.

target element with leading edge, but with much higher peak value at the hottest line, which results in an extreme toroidal asymmetry. The maximum surface temperature rises to $3397 \mathrm{~K}$ at $10 \mathrm{~s}$ at the hottest end, while a sharp drop of the temperature after $10 \mathrm{~s}$ is due to the additional thermal diffusion toroidally towards the colder end, as shown in Figure A.6. No carbon sublimation process or radiation is considered in the temperature evolution for the 3D model. In the $2 \mathrm{D}$ code, this toroidal diffusion channel is not considered, such that the faster decrease of the temperature is falsely interpreted as an artificially negative heat flux from the top after $10 \mathrm{~s}$. It is clear that at the leading edge end the inversely calculated heat flux is not valid. However, the leading edge effect seems to be negligible for the central line of the finger, as shown in Figure A.7. This is because of the much slower thermal diffusion toroidally due to the smaller toroidal temperature gradient compared with radial and perpendicular directions. In Figure A.8 the calculated heat flux profiles of the hottest line, the central line as well as the averaged line (averaging method described in section 5) are compared. While the central heat flux profile is almost the same as case 1 , the averaged profile is influenced by the artificially high heat flux calculated from the hotter region, which increases the averaged peak heat flux by $\sim 14 \%$ from $3.5 \mathrm{MW} \mathrm{m}^{-2}$ to $4 \mathrm{MW} \mathrm{m}^{-2}$.

From the analysis, it can be concluded that the castellation structures have a small impact on the radial heat diffusion, and the 2D model can reproduce the input heat flux with an acceptable deviations. The calculated heat flux profile is slightly broader than the real input with an underestimation of the peak heat load up to $\sim 8 \%$ given a typical pulse length of $10 \mathrm{~s}$ in OP1.2. However, in the experiments, the jumps of the calculated heat flux between two nearby tiles are not obvious. This is due to the increased heat exchange contributed from thermal radiation in between the castellations 
(only $0.8 \mathrm{~mm}$ gap), which would more or less alleviates the general thermal barriers caused by them.

For the toroidally asymmetric power loads caused by the leading edges, the heat fluxes calculated at the edge of the finger are not trustful. For dedicated leading edge analysis, one should apply the interpolated lines of temperature for the comparison with modellings. On the other hand, different from the extreme case shown above, in the experiments the leading edge effects are less severe, with typically smaller $h_{\mathrm{LE}}$ on the order of $0.2 \mathrm{~mm}$ [21], as well as radiation being considered. The dominant temperature gradients are still in the tile depth and radial directions as implemented in the $2 \mathrm{D}$ code. For magnetic topology studies, the central line of the finger would provide reliable heat flux profile, even for the most prominent leading edges. An average over all the heat flux profiles on a finger with small toroidal variation of footprint is also acceptable, aiming for a better statistics for the the strike line shape. In contrast to the case demonstrated above, such averaging applied on the nearby finger with a shadowed edge would results in an opposite lowered heat flux compared with the input from the top. The integral power loads over the whole target is considered to be valid, due to the balancing of the effects between the leading and the corresponding shadowed edges.

\section{References}

[1] Klinger, T., AlOnso, A., BOZHenkOV, S., et al., Plasma Physics and Controlled Fusion 59 (2017) 014018.

[2] SUnN PEDERSEn, T., DINkLAGE, A., TURKIN, Y., et al., Physics of Plasmas 24 (2017) 055503.

[3] WOlf, R., Ali, A., AlOnsO, A., et al., Nuclear Fusion 57 (2017) 102020 .

[4] FENG, Y., SARDEI, F., GRIGULL, P., et al., Nuclear Fusion 46 (2006) 807.

[5] PEDERSEN, T. S., KNIG, R., KRYCHOWIAK, M., et al., Plasma Physics and Controlled Fusion 61 (2019) 014035.

[6] JAKUBOWSKI, M., ALI, A., DREWELOW, P., et al., 27th IAEA Fusion Energy Conference (2018).

[7] BOZHENKOV, S., GEIGER, J., GRAHL, M., et al., Fusion Engineering and Design 88 (2013) 2997 . 
[8] HERRMANN, A., JUNKER, W., GUNTHER, K., et al., Plasma Physics and Controlled Fusion 37 (1995) 17.

[9] JAKUBOWSKI, M., EVANS, T., FENSTERMACHER, M., et al., Nuclear Fusion 49 (2009) 095013.

[10] AHN, J.-W., MAINGi, R., MASTROVito, D., and ROQUEMORE, A. L., Review of Scientific Instruments 81 (2010) 023501.

[11] TEMmerman, G. D., DELCHAmBRE, E., DOWLING, J., et al., Plasma Physics and Controlled Fusion 52 (2010) 095005.

[12] HUBER, A., KINNA, D., HUBER, V., et al., Physica Scripta 2017 (2017) 014027.

[13] BAlBOA, I., ARNOUX, G., EICH, T., et al., Review of Scientific Instruments 83 (2012) 10D530.

[14] JAKUBOWSKI, M., DREWELOW, P., FELLINGER, J., et al., Review of Scientific Instruments 89 (2018) 10E116.

[15] PUIG SITJES, A., JAKUBOWSKI, M., ALI, A., et al., Fusion Science and Technology 74 (2018) 116.

[16] ALI, A., JAKUBOWSKI, M., GREUNER, H., et al., Physica Scripta 2017 (2017) 014074.

[17] EICH, T., THOMSEN, H., FUNDAMENSKI, W., et al., Journal of Nuclear Materials 415 (2011) S856 , Proceedings of the 19th International Conference on Plasma-Surface Interactions in Controlled Fusion.

[18] FENG, Y., KOBAYASHI, M., LUNT, T., and REITER, D., Plasma Physics and Controlled Fusion 53 (2011) 024009.

[19] LAZERSON, S. A., OTTE, M., JAKUBOWSKI, M., et al., Nuclear Fusion 57 (2017) 046026.

[20] BOZHENKOV, S., JAKUBOWSKI, M., NIEMANN, H., et al., Nuclear Fusion 57 (2017) 126030.

[21] ENDLER, M., FELLINGER, J., HLBE, H., et al., Plasma Physics and Controlled Fusion 61 (2019) 025004.

[22] GEIGER, J., BEIDLER, C. D., FENG, Y., et al., Plasma Physics and Controlled Fusion 57 (2015) 014004. 
[23] PISANO, F., CANNAS, B., JAKUBOWSKI, M. W., et al., Review of Scientific Instruments 89 (2018) 123503.

[24] HILLE, E., Analytic function theory, volume 2, American Mathematical Soc., 2005.

[25] DELAUnAY, B., Bulletin de l'Académie des Sciences de l'URSS. Classe des sciences mathématiques et na 6 (1934) 793. 